\title{
SIMS zircon ages and Nd isotope systematics of the 2.2 Ga mafic intrusions in northern and eastern Finland
}

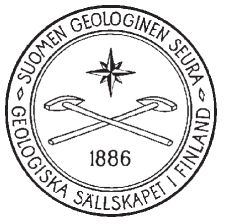

Eero Hanski ${ }^{1) *}$, Hannu Huhma ${ }^{2)}$ and Jouni Vuollo ${ }^{3)}$

1) Department of Geosciences, P.O. Box 3000, FI-90014 University of Oulu, Finland

2) Geological Survey of Finland, P.O. Box 96, FI-02151 Espoo, Finland

3) Geological Survey of Finland, P.O. Box 77, FI-96101 Rovaniemi, Finland

\begin{abstract}
Using the SIMS, ID-TIMS and Sm-Nd isotopic methods and the electron microprobe, we have studied several differentiated mafic intrusions of the c. $2.2 \mathrm{Ga}$ gabbro-wehrlite association (GWA) from four Paleoproterozoic schist belts and the Archean Kuhmo Greenstone Belt. Back-scattered electron images and electron microprobe analyses revealed that zircon crystals vary from well-preserved to turbid and highly altered with individual grains often displaying irregular, hydrated, CaO-bearing domains. In the most pristine domains, suitable for establishing the crystallization ages, SIMS ${ }^{207} \mathrm{~Pb} /{ }^{206} \mathrm{~Pb}$ ages fall in the range of 2210-2220 Ma, which is consistent with the most concordant ID-TIMS U$\mathrm{Pb}$ ages. One of the studied intrusions that had previously yielded a conventional U-Pb date of less than $2.0 \mathrm{Ga}$, could be shown by spot analysis to belong to the $2.2 \mathrm{Ga}$ family. In contrast to the well-preserved zircon domains, altered domains exhibit a variable and often strong $\mathrm{U}-\mathrm{Pb}$ discordance up to $70 \%$ and have distinctly lower ${ }^{207} \mathrm{~Pb} /{ }^{206} \mathrm{~Pb}$ ages. Some zircon grains record isotopic resetting at the time of the Svecofennian orogeny (ca. 1.8-1.9 Ga), while the most discordant ones project in the concordia diagram to late Paleozoic lower intercept ages indicating a relative recent $\mathrm{Pb}$ loss. The mineral chemistry of zircon suggests that the leakage of radiogenic $\mathrm{Pb}$ can be ascribed to an opensystem behavior related to hydrothermal alteration via action of $\mathrm{CaCl}_{2}$-bearing fluids.
\end{abstract}

Common albitization of plagioclase in the GWA intrusions has caused this mineral to behave as an open system with regard to the Sm-Nd isotopic systematics. Despite this uncertainty, our $\mathrm{Nd}$ isotopic data indicate that the magma that produced the GWA intrusions in various parts of northern and eastern Finland was isotopically homogeneous and had an initial $\varepsilon_{\mathrm{Nd}}(2220 \mathrm{Ma})$ value of c. +0.6 precluding significant upper crustal contamination upon emplacement and subsequent fractional crystallization.

Key words: intrusions, gabbros, granophyre, absolute age, U/Pb, Sm/Nd, zircon, electron probe data, Paleoproterozoic, Northern Finland, Eastern Finland

* Corresponding author email: eero.hanski@oulu.fi 


\section{Introduction}

One of the distinct phases of the Paleoproterozoic mafic magmatism in Finland is represented by the c. 2.2 Ga layered sills, which are widely spread in eastern and northern Finland (Vuollo \& Huhma, 2005). On the basis of the predominant rock types, Hanski (1986a, b, 1987) assigned these intrusive rocks to the gabbro-wehrlite association (GWA). Later the rocks have also been called karjalites (Vuollo \& Piirainen, 1992).

Since the beginning of the 1970s, coarse-grained or pegmatoidal gabbros in the upper parts of the GWA sills have served as favorable targets for dating purposes as they contain abundant zircon (Sakko, 1971; Kouvo, 1977). Occasionally, baddeleyite has also been found in these rocks (e.g. Hyppönen, 1983; Perttunen \& Vaasjoki, 2001). Hanski et al. (2001) compiled available ID-TIMS U-Pb analyses of zircon and baddeleyite from the dated GWA sills from eastern and northern Finland. Even though the apparent ID-TIMS ages of the dated intrusions have a spread of more than $200 \mathrm{Ma}$, they concluded that the time interval of the magmatic event that produced these rocks was probably considerably shorter. The reason for this ostensible discrepancy is the fact that analytical data from individual dating sites often display a considerable discordance on concordia diagrams (see figure 2 in Hanski et al., 2001) and therefore the interpretation of the isotopic results has not always been straightforward. Under the microscope, zircon crystals from the GWA intrusions commonly display a very turbid appearance indicative of a high degree of metamictization, and conventional ID-TIMS studies have revealed that they often have high $\mathrm{U}$ contents (e.g. Perttunen \& Vaasjoki, 2001; Hanski et al., 2001).

The high sensitivity and high spatial resolution of the ion microprobe provides a powerful tool for in situ dating of single zircon grains (e.g. Williams, 1998). By analysis of different domains within zircon crystals, this method allows primary crystallization ages to be determined also for the zircon populations that have experienced various degrees of radiation damage and subsequent partial open- ing of the U-Pb isotopic system. Six samples from northern and eastern Finland, five gabbros and one granophyre, which differ from each other in terms of the degree of post-magmatic alteration and the geological environment, were selected for in situ zircon dating at the NORDSIM facility in Stockholm. Zircon and/or baddeleyite in all these samples were previously dated using the conventional multi-grain method of isotope dilution thermal ionization mass spectrometry (ID-TIMS). We also report ID-TIMS $\mathrm{U}-\mathrm{Pb}$ zircon data from two locations. In addition, we used an electron microprobe to see how different domains within zircon grains, as revealed by back-scattered electron (BSE) images, deviate chemically from each other. Only a limited number of $\mathrm{Nd}$ isotopic data have so far been published from the GWA intrusions (Huhma et al., 1990; Huhma et al., 1996). In this paper, we present new $\mathrm{Nd}$ isotopic data from several intrusions.

\section{General characteristics of the GWA sills}

Sill-like, gravity-differentiated intrusions of the gabbro-wehrlite association have been found in Karelian schist belts (Northern Karelia, Kainuu, Kuusamo, Peräpohja, Central Lapland) in eastern and northern Finland. Typically the sills occur close to the unconformity between the Archean granitegneiss basement and overlying Paleoproterozoic sedimentary and volcanic rocks, usually intruding concordantly into "Jatulian" quartzitic metasediments but occasionally shifting their position to the underlying basement (Vuollo \& Huhma, 2005).

Individual sills may reach several hundred meters in thickness and they can often be traced for many kilometers along strike. The maximum length of more than $100 \mathrm{~km}$ is attained by the Runkaus sill in the Peräpohja Belt. From the bottom upwards, the layered sequence of the sills normally comprises cumulates with the following cumulus mineral assemblages: olivine, olivine-clinopyroxene, clinopyroxene, clinopyroxene-magnetite and plagioclase-clinopyroxene-magnetite. Orthopyroxene may be present in the olivine-clinopyroxene cumulates, but its abundance is always low. The ultramafic 
cumulates characteristically contain magmatic edenitic hornblende as poikilitic grains. Plagioclase in gabbroic cumulates is commonly altered to secondary albite, but more calcic ones with An up to 47 $\%$ have been discovered in some intrusions in Lapland (Hanski, 1987). The parental magma of the GWA intrusions corresponded to hydrous, low-Al tholeiite (Vuollo \& Piirainen, 1992), which explains the late appearance of plagioclase as a cumulus phase. The magma was characterized by a low $\mathrm{Al}_{2} \mathrm{O}_{3} / \mathrm{TiO}_{2}$ ratio (5-6) and differed in this respect from other "Jatulian" mafic magmas. It was also enriched in incompatible elements and had LREEenriched chondrite-normalized REE patterns with $(\mathrm{La} / \mathrm{Yb})_{\mathrm{CN}}$ of -4-5 (Hanski, 1986a).

\section{Description of sampling sites and samples}

Previously one GWA intrusion from the Kuusamo Schist Belt has been dated using the SIMS method (Evins \& Laajoki, 2001). For our U-Pb and Sm$\mathrm{Nd}$ study, we chose samples from intrusions occurring in four other Paleoproterozoic supracrustal belts in northern and eastern Finland: the Central Lapland Greenstone Belt, Peräpohja Schist Belt, Tahkomäki-Kinahmi schist belt, and North Karelian Schist Belt. In addition, we sampled c. 2.2 Ga intrusions found within the Archean Kuhmo Greenstone Belt. The sampling sites are shown in Fig. 1 and described below in the order of their geographical position from north to south.

\subsection{Silmäsvaara, Haaskalehto, Ahvenvaara}

There is a string of mafic-ultramafic bodies close to the southern margin of the Central Lapland Greenstone Belt (Fig. 1). Their occurrence can be easily discerned on geophysical maps due to the presence of magnetite-bearing gabbros and variably altered ultramafic cumulates in these bodies. Among them are the Silmäsvaara, Haaskalehto and Ahvenvaara intrusions, which are included in the present study. It is possible that these three intrusions originally belonged to the same sill-like intru- sive body, which was injected concordantly into sedimentary rocks of the Sodankylä Group and was later disrupted into separate blocks due to tectonic movements (Hanski \& Huhma, 2005).

The Haaskalehto intrusion is situated $-20 \mathrm{~km}$ west of Sodankylä. It can be regarded as the type occurrence of the GWA in central Finnish Lapland (Lehtonen et al., 1998). On aeromagnetic maps, the Haaskalehto intrusion can be followed along strike for c. $5 \mathrm{~km}$ and its thickness is estimated to be c. $0.5 \mathrm{~km}$. The rock sequence is reasonably well exposed and comprises wehrlites, pyroxenites and gabbros. Despite the fact that the sill is in contact with an intrusive granite on its southern side, the original magmatic mineralogy is usually well preserved (e.g. olivine $\mathrm{Fo}_{72.6-81.6}$, plagioclase up to $\mathrm{An}_{47}$; Hanski, 1987).

The Haaskalehto intrusion was chosen for this study because its zircon grains are known to belong to the least altered ones that have been encountered in the GWA intrusions. Tyrväinen (1983) reported a concordant ID-TIMS U-Pb zircon age of $2220 \pm$ $11 \mathrm{Ma}$ for the gabbro sample A892. The same sample was used for SIMS zircon analyses in this study. In addition, we utilized three gabbroic samples, A1408, 19.1-HSP-78, and 24-HSP-78, together with separated minerals (plagioclase, clinopyroxene, amphibole) for Sm-Nd isotopic analyses.

The eastern end of the Silmäsvaara intrusion is located c. $5 \mathrm{~km}$ WNW of the western end of the Haaskalehto intrusion. The sill is c. $5 \mathrm{~km}$ long and more than $0.5 \mathrm{~km}$ thick. The gabbroic rocks are not as fresh as those at Haaskalehto, but the olivine pyroxenites in the lower part of the intrusion contain portions with original magmatic minerals still abundantly present. Sampling for this study was restricted to one olivine-bearing pyroxenite sample, A1430, from which plagioclase and pyroxene were separated for $\mathrm{Sm}-\mathrm{Nd}$ isotopic analysis.

The Ahvenvaara intrusion is located on the southern side of the Pyhätunturi Mountain c. $50 \mathrm{~km}$ SSE of Sodankylä. On magnetic maps, it has an appearance of a $2 \times 3 \mathrm{~km}$ block that has been rotated by c. $90^{\circ}$ relative to the general strike of the surrounding metasedimentary rocks. The exposed rock types of the intrusion are limited to olivine pyroxe- 


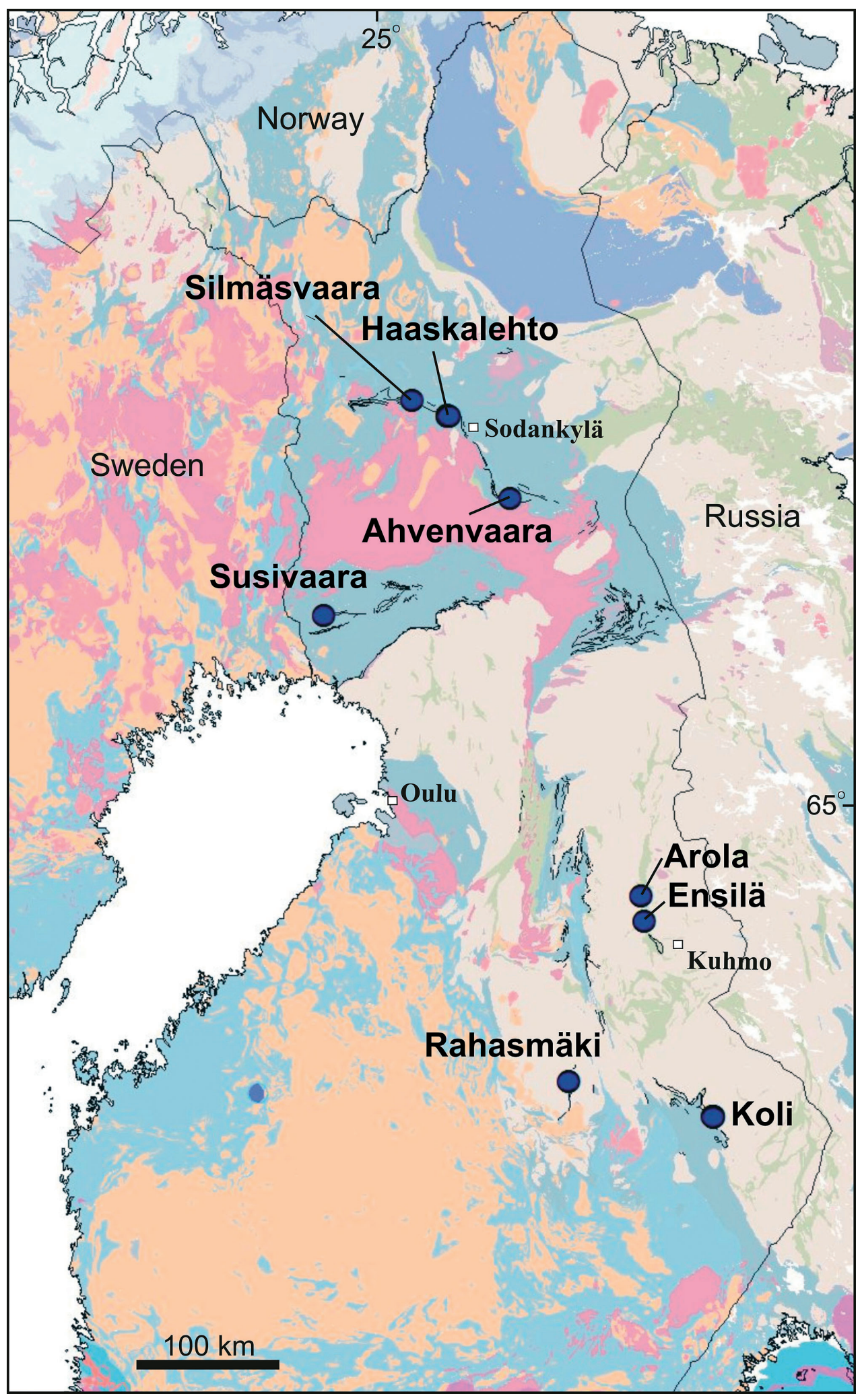

Fig. 1. Map showing sampling sites in northern and eastern Finland. 2.2 Ga layered sills are shown as black lines. The base map modified after Koistinen et al. (2001). 
nites and pyroxenites. The Ahvenvaara intrusion provided a fresh olivine pyroxenite sample, A1431, and plagioclase and pyroxene separates for our $\mathrm{Sm}-$ $\mathrm{Nd}$ isotope study. It is worth mentioning that Räsänen and Huhma (2001) published a precise IDTIMS U-Pb zircon age of $2222 \pm 6 \mathrm{Ma}$ for the Harjunoja intrusion, which is a GWA-type sill located geographically between the Haaskalehto and Ahvenvaara intrusions.

\subsection{Susivaara}

From the Peräpohja Schist Belt 14 samples have earlier been used for conventional U-Pb dating of the gabbro-wehrlite association (Perttunen \& Vaasjoki, 2001). For this study we chose one of these samples, A865 from Susivaara, which was known to display a significant spread in discordance between separate zircon fractions. The Susivaara sill is located in the western part of the Peräpohja Schist Belt, on the Törmäsvaara map sheet described by Perttunen and Hanski (2003). It forms a c. 100-mthick, weakly differentiated gabbroic body intruded concordantly into quartzites and siltstones of the Palokivalo Formation of the Kivalo Group. The same metasediment unit hosts a larger, more typical layered GWA body, the Kivimaa sill, which has also been dated (see below).

Earlier $\mathrm{U}-\mathrm{Pb}$ isotope studies on the Susivaara intrusion have revealed that zircon and baddeleyite are discordant whereas sphene is concordant (Perttunen \& Vaasjoki, 2001). Two concordant analyses on sphene together with three discordant analyses of baddeleyite ( \pm zircon intergrowths) provided an upper intercept age of $2208 \pm 19$ Ma. Perttunen and Vaasjoki (2001) reported similar results for the nearby Kivimaa sill: sphene yielded a concordant $\mathrm{U}$ - $\mathrm{Pb}$ age of $2216 \pm 8 \mathrm{Ma}$, while zircon and baddeleyite fractions were discordant though close to a chord and indicated an age of c. 2215 Ma.

\subsection{Rahasmäki}

The Rahasmäki sill is located in the northern part of the Tahkomäki-Kinahmi schist belt, which is a small, 1.0-3.5-km-wide and c. 40-km-long, NS- trending Karelian supracrustal belt surrounded by Archean gneisses (Paavola, 1984). There are only a few outcrops, but aeromagnetic data show that on the present erosion surface, the intrusion forms a folded, U-shaped (synclinal), 100-200-m-thick body emplaced concordantly into quartzites or occurring partly in the Archean basement. The exposed parts consist of coarse-grained metagabbro and minor metapyroxenite.

Paavola (1984) published five U-Pb analyses on zircon from a gabbroic sample, which yielded concordia intercepts at $1967 \pm 24 \mathrm{Ma}$ and $425 \pm 48$ Ma. However, the data do not plot exactly on a chord (MSWD = 30), which together with the geological setting of this intrusion and its lithological characteristics resembling those of the GWA intrusions has caused some uncertainty on the real crystallization age of the intrusion. In order to clarify this question, we analyzed zircon grains by SIMS from the same sample, A977, as employed by Paavola (1984).

\subsection{Ensilä}

The presence of Paleoproterozoic mafic intrusions within the Archean Kuhmo Greenstone Belt was indicated for the first time by Hyppönen (1983) who published age determinations of two mafic dikes from this belt. However, the discordance of the results precluded the calculation of precise crystallization ages. Later Hanski (1982, 1984) performed a comparative study of the intrusive mafic-ultramafic rocks in the Kuhmo Greenstone Belt and the "Jatulian albite diabases" in the Koli area and came to the conclusion that many "Jatulian" mafic intrusive bodies can be found within the Kuhmo Greenstone Belt. For example, a continuous differentiation series from amphibole-bearing olivine cumulates to coarse-grained gabbros, analogous of that displayed by the Koli sill, can be observed near the Ensilä farm, c. $30 \mathrm{~km} \mathrm{NW}$ of Kuhmo. In addition, the GWA magmatism has generated flow-differentiated mafic-ultramafic dikes within the greenstone belt (Hanski, 1984). In the Ensilä area, the originally horizontal cumulate layering of the gravitydifferentiated bodies dip now by an angle of c. $45^{\circ}$ 
demonstrating Paleoproterozoic folding of these intrusions and the associated Archean greenstone belt as well. It is peculiar that GWA intrusions have been encountered within the relatively narrow, NS trending greenstone belt, but so far not in the adjacent granitoid basement outside the greenstone belt.

Lithological and geochemical data on the GWA sills in the Ensilä area can be found in Hanski (1984). The sills are spatially associated with the Kellojärvi Ultramafic Complex (Tulenheimo, 1999). This complex was earlier thought to belong to the GWA (Hanski, 1984), but was later shown to be Archean in age and is probably related to the komatiitic magmatism of the greenstone belt ( $\mathrm{Pa}$ punen et al., 1998).

A coarse-grained metagabbro sample, A586, was used for conventional zircon dating. The rock is moderately altered; for example, ilmenomagnetite is replaced partly by biotite and there are small garnet crystals enclosed in plagioclase. The same sample was also used for SIMS dating. Sm-Nd isotopic results on GWA rocks from Kuhmo have previously been reported by Tulenheimo (1999) and are included in our Table 4. They contain eight wholerock analyses of wehrlites, metapyroxenites and metagabbros from the Ensilä area and one wehrlite (TTT-170-96) and its two pyroxene separates from the Arola area, c. $15 \mathrm{~km}$ north of Ensilä.

\subsection{Koli}

One of the best-exposed GWA sills in Finland can be found in the Koli area, Northern Karelia. Detailed descriptions of the stratigraphic sections and geochemical and mineralogical data of the Koli sill have been published from different localities (Piirainen, 1969; Hanski, 1982, 1984; Vuollo \& Piirainen, 1992). The sill can be followed for more than $60 \mathrm{~km}$ along strike and has a maximum thickness of $340 \mathrm{~m}$. It is partly located in the Archean basement following conformably the contact between the Archean rocks and the overlying Jatulian quartzites and partly emplaced into these quartzites. It cuts the two lowermost sedimentary lithostratigraphic units, the Koli Formation and Jero Formation, but has not been observed to cut the uppermost Jatulian formation, the Puso Formation (Piirainen \& Vuollo, 1991). Relatively good exposure has facilitated determination of the detailed stratigraphy of the Koli sill. From the bottom upwards, the following zones have been recognized: wehrlite, clinopyroxenite, magnetite clinopyroxenite, magnetite gabbro, coarse-grained gabbro, granophyre, clinopyroxenite (the upper marginal zone) (Vuollo \& Piirainen, 1992).

Sampling for isotopic analysis was performed at a segment of the Koli sill that was emplaced into Archean gneisses. Three samples from three different localities were utilized: samples A587 and A1182 from the main gabbroic cumulate at Savilahti and Kaunislahti, respectively, and sample A1096 from Kaunisniemi, representing granophyre from the most evolved part of the sill. Baddeleyite was analyzed using ID-TIMS from sample A587 and zircon from samples A1096 and A1182. Zircon grains from the latter two samples were also studied using the NORDSIM facility.

Five samples from the Koli sill were analyzed for $\mathrm{Sm}-\mathrm{Nd}$ isotopes. Whole-rock analyses were performed for two gabbro samples, 48-JIV-85 and A1182, and one granophyre sample labelled A1096a. In addition, $\mathrm{Sm}-\mathrm{Nd}$ isotopic compositions were determined on plagioclase and pyroxene separates from two clinopyroxenite samples, A1220 and A1221.

\section{Analytical methods}

\subsection{U-Pb isotopic analyses}

Procedures for conventional U-Pb analyses followed the method by Krogh (1973) and involved aliquoting the $\mathrm{HCl}$ solution and addition of ${ }^{208} \mathrm{~Pb} /$ ${ }^{235} \mathrm{U}$ isotopic tracer. Measurements were made using a VG Sector 54 mass spectrometer at the Geological Survey of Finland (GSF), Espoo. The performance of the ion counter was checked by repeated measurements of a NBS 983 standard.

In situ U-Th- $\mathrm{Pb}$ analyses of zircons were carried out using a Cameca IMS1270 ion microprobe at the Swedish Museum of Natural History, Stockholm (the NORDSIM facility). The analytical pro- 
cedure is described in Whitehouse et al. (1997, 1999). The spot diameter for the $4 \mathrm{nA}$ primary $\mathrm{O}_{2}$ ion was c. $30 \mathrm{~mm}$, and oxygen flooding was used to improve the ionization of $\mathrm{Pb}$. Calibration of the $\mathrm{U} /$ $\mathrm{Pb}$ ratio was based on analyses of the Geostandards zircon 91500, which has an age of $1065 \mathrm{Ma}$ (Wiedenbeck et al., 1995). Correction of the measured isotopic ratios for common $\mathrm{Pb}$ was estimated from monitored ${ }^{204} \mathrm{~Pb}$ counts and the terrestrial average $\mathrm{Pb}$ isotopic composition at $1900 \mathrm{Ma}$ was used for this correction (Stacey \& Kramers, 1975). Data reduction was performed using the NORDSIM software written by Martin Whitehouse, and data regressions were carried out using the Isoplot/Ex 2.49 program of Ludwig (2001). Back-scattered electron images were used to select targets for SIMS analyses.

\subsection{Sm-Nd isotopic analyses}

The Sm-Nd isotopic work was performed at the GSF on a VG Sector 54 mass spectrometer using the freshest samples available. Standard procedures were used for crushing and separation of plagioclase and pyroxene with final purification made by hand-picking when necessary. For Sm-Nd analyses, mineral concentrates were washed ultrasonically in warm $6 \mathrm{~N} \mathrm{HCl}$ for $30 \mathrm{~min}$ and rinsed several times in water. The samples (150-200 mg) were dissolved in $\mathrm{HF}-\mathrm{HNO}_{3}$ using Savillex screw cap teflon beakers or sealed teflon bombs (felsic whole rocks) for $48 \mathrm{~h}$. Mixed ${ }^{149} \mathrm{Sm}-{ }^{150} \mathrm{Nd}$ spike was added to the sample prior the dissolution. After careful evaporation of fluorides, the residue was dissolved in $6 \mathrm{~N} \mathrm{HCl}$ and a clear solution was achieved. Samarium and $\mathrm{Nd}$ were separated in two stages using a conventional cation exchange procedure $(7$ $\mathrm{ml}$ of AG50Wx8 ion exchange resin in a bed of 12 $\mathrm{cm}$ length) and a modified version of the TeflonHDEHP (hydrogen diethylhexyl phosphate) method developed by Richard et al. (1976). The measurements have been made in a dynamic mode using Ta-Re triple filaments. ${ }^{143} \mathrm{Nd} /{ }^{144} \mathrm{Nd}$ ratio is normalized to ${ }^{146} \mathrm{Nd} /{ }^{144} \mathrm{Nd}=0.7219$. The average value for the La Jolla standard is ${ }^{143} \mathrm{Nd} /{ }^{144} \mathrm{Nd}=0.511850$ $\pm 7(1 \sigma, \mathrm{n}=70$, triple filament measurements du- ring 1995-2001). The $\mathrm{Sm} / \mathrm{Nd}$ ratio of the spike was calibrated against the Caltech mixed $\mathrm{Sm} / \mathrm{Nd}$ standard (Wasserburg et al., 1981). Based on duplicated analyses, the error in ${ }^{147} \mathrm{Sm} /{ }^{144} \mathrm{Nd}$ is estimated to be $0.4 \%$. Initial ${ }^{143} \mathrm{Nd} /{ }^{144} \mathrm{Nd}$ ratios and $\varepsilon_{\mathrm{Nd}}$ values were calculated with the following parameters: $\lambda{ }^{147} \mathrm{Sm}=6.54 \times 10^{-12} \mathrm{a}^{-1},{ }^{147} \mathrm{Sm} /{ }^{144} \mathrm{Nd}=0.1966$ and ${ }^{143} \mathrm{Nd} /{ }^{144} \mathrm{Nd}=0.51264$ for present CHUR. Depleted mantle model ages (T-DM) were calculated according to DePaolo (1981). Measurement on the rock standard BCR-1 provided the following values: $S \mathrm{~m}=6.58 \mathrm{ppm}, \mathrm{Nd}=28.8 \mathrm{ppm},{ }^{147} \mathrm{Sm} /$ ${ }^{144} \mathrm{Nd}=0.1380,{ }^{143} \mathrm{Nd} /{ }^{144} \mathrm{Nd}=0.51264 \pm 0.00002$. The blank measured during analyses was: $30-100$ pg for Sm and 100-300 pg for Nd. Programs by Ludwig $(1991,2001)$ have been employed for age calculations.

A few older $\mathrm{Sm}-\mathrm{Nd}$ analyses measured using an old technique and a non-commercial mass spectrometer (Huhma, 1986) are included in this paper. Compared with more recent analyses, they tend to yield slightly larger errors in ${ }^{143} \mathrm{Nd} /{ }^{144} \mathrm{Nd}$, but based on duplicated newer analyses, are consistent within error.

\subsection{Electron microprobe analyses}

In order to acquire preliminary data on the relationship between zircon chemistry and the observed U-Pb isotopic and BSE characteristics, different domains of a zircon grain were analyzed for major ( $\mathrm{Zr}, \mathrm{Si}$ ) and trace (Hf, Y, Ca, Fe, Mn, U, Th, $\mathrm{Pb}$ ) elements. Electron microprobe analyses were performed by the wavelength dispersive technique using a Cameca SX100 microprobe at the Geological Survey of Finland in Espoo. The analytical conditions were an accelerating potential of $20 \mathrm{keV}$, a sample current of $30 \mathrm{nA}$, and a beam diameter of 1 $\mu \mathrm{m}$. Synthetic cubic zirconia was employed as the standard for Zr, Y, and Hf, while natural galena was used for $\mathrm{Pb}$, diopside for $\mathrm{Si}$ and $\mathrm{Ca}$, rhodonite for $\mathrm{Mn}$, and almandine for Fe. The uranium and thorium standards were pure metals. Back-scattered electron images were obtained with a JEOL JCXA733 scanning electron microscope (SEM) at the Department of Electron Optics, University of Oulu. 


\section{Results}

\subsection{U-Pb geochronological results}

Ion microprobe $\mathrm{U}-\mathrm{Th}-\mathrm{Pb}$ analytical data for zircons are listed in Table 1 and plotted on concordia diagrams in Figs. 3, 6, 8, 10 and 13. In these figures, the error boxes represent $2 \sigma$ errors of isotope ratios and the uncertainties in the calculated ages are reported at the $95 \%$ confidence level. New conventional ID-TIMS data are presented in Table 2 and, together with the previously published ID-TIMS data from the studied samples, are compared with SIMS results in the above mentioned concordia diagrams.

\subsubsection{Haaskalehto (A892)}

The Haaskalehto intrusion represents one of the rare locations of the GWA intrusions from which previous conventional $\mathrm{U}-\mathrm{Pb}$ analyses on zircon plot relatively close to the concordia curve and thus pro- vide a reliable age estimate $(2220 \pm 11 \mathrm{Ma}$; Tyrväinen, 1983). This is in harmony with the good preservation of the zircon grains that is evident from the BSE images taken of zircons from sample A892. As shown in Fig. 2, the grains have a weak oscillatory zoning and are remarkably clean without darker, patchy alteration patterns displayed by metamict zircons in the samples from the other study areas (see below).

Four $\mathrm{U}-\mathrm{Pb}$ analyses on four zircon grains made by ion microprobe are all concordant and give an age of $2211 \pm 6 \mathrm{Ma}$ (Fig. 3) which overlaps the age obtained by the conventional method. The analyses show a low common lead but a fairly large range in $\mathrm{Th} / \mathrm{U}$ ratio (Table 1 ).

\subsubsection{Susivaara (A865)}

In terms of alteration, the studied zircon grains from the Susivaara intrusion represent an extreme case. Most of them are very dark brown and turbid. Backscattered electron images show complex alteration

\section{A892 Haaskalehto}
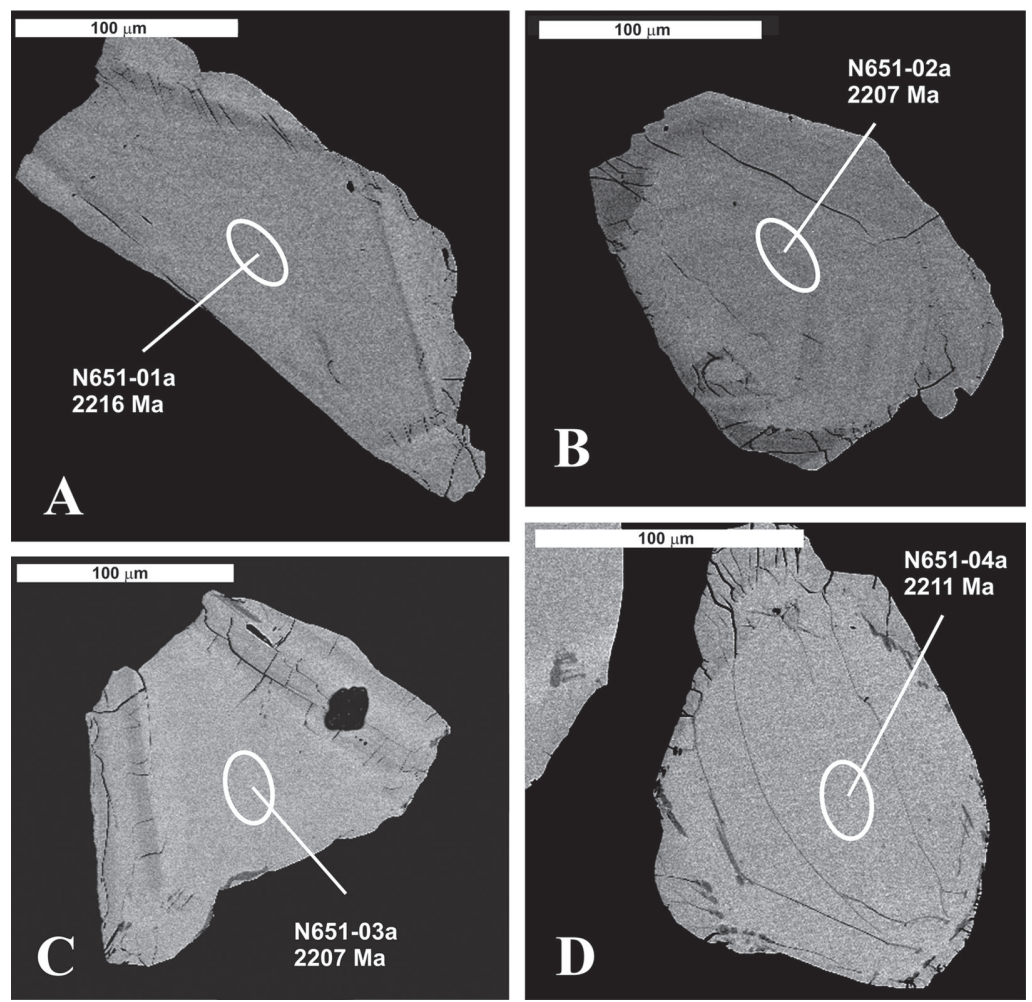

Fig. 2. BSE images of zircon grains from the Haaskalehto intrusion (sample A892). Spots analyzed by ion microprobe are shown as white ellipsoids. All annotated ages are ${ }^{207} \mathrm{~Pb} /{ }^{206} \mathrm{~Pb}$ ages, which may differ from upper intercept ages depending on the degree of discordance. 


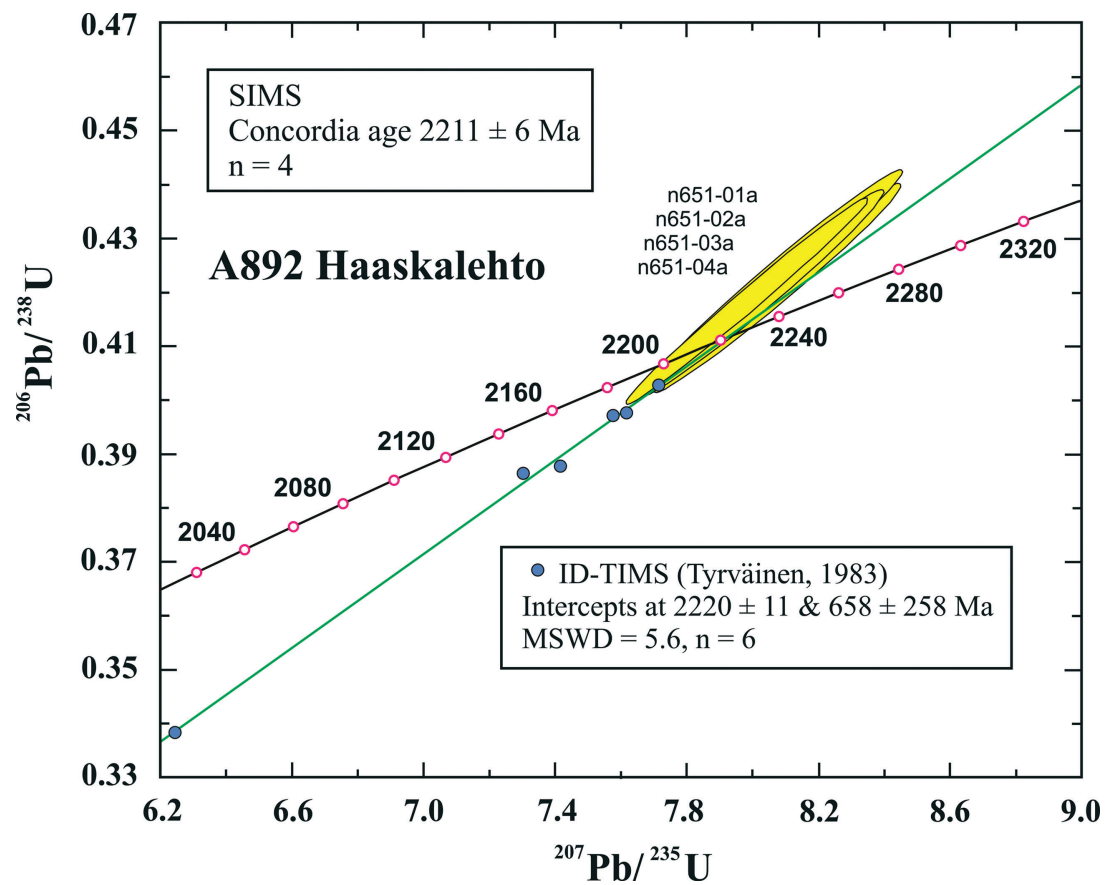

Fig. 3. Concordia plots of U-Pb data of sample A892 from the Haaskalehto intrusion, Central Lapland. SIMS analyses shown as yellow error ellipsoids and ID-TIMS analyses by blue circles without error indication. Note that the scale is different from the other concordia diagrams. features in most zircon crystals. The altered parts of the grains are characterized by a lowered BSE intensity, i.e. a lower mean atomic number with respect to the apparently unaltered domains (Fig. 4). The BSE contrast between altered and more pristine domains is often obvious (for example, grains n654-02 and n654-03 in Figs. 5 and 4B). Even using the largest magnification of SEM, the darker, grayish domains, such as shown in Fig. 4A, appear homogeneous in BSE images without recognizable intergrowths of zircon and other phases. Some zircon crystals are completely altered to heterogeneous, spongy material (Fig. 4E). The altered domains often contain tiny thorite grains standing up as white specks in BSE images (see below).

Twelve conventional U-Pb analyses on the Susivaara sample A865 published previously by Perttunen and Vaasjoki (2001) are shown in Fig. 6. They are widely scattered. The cleaner zircon fractions and baddeleyite separates plot closer to the concordia curve than the turbid zircon fractions. Two sphene analyses are nearly concordant. Perttunen and Vaasjoki (2001) calculated an age of $2208 \pm 19 \mathrm{Ma}$ using baddeleyite and sphene fractions. Including discordant zircon fractions does not change the age but increases the error (2208 \pm 29 Ma, see Fig. 6). Eleven analyses on eight zircon grains were obtained by ion microprobe. The data are technically good but scattered and discordant (Fig. 6). Interestingly, the well-preserved zircon domains have isotopic compositions relatively close to the concordia curve with ${ }^{207} \mathrm{~Pb} /{ }^{206} \mathrm{~Pb}$ ages of c. $2.2 \mathrm{Ga}$ (n654-02a and n654-03a, Fig. 6), whereas the altered zircon zones exhibit strong $\mathrm{U}-\mathrm{Pb}$ discordance with lower ${ }^{207} \mathrm{~Pb} /{ }^{206} \mathrm{~Pb}$ ages (ca. 1.7-2.0 Ga) n654-02b and n654-03b, Fig. 6). The U-Th-Pb data show that the altered domains tend to be enriched in Th. For example, in analysis $\mathrm{n} 654-02 \mathrm{~b}$ the $\mathrm{Th} / \mathrm{U}$ ratio is 11 (Table 1).

The five most pristine domains provide an upper intercept age of $2198 \pm 24 \mathrm{Ma}$ (lower intercept at $154 \pm 230 \mathrm{Ma}, \mathrm{MSWD}=4.2$, Fig. 6), which can be considered the best estimate for magmatic crystallization. The data on this sample do not constrain the age of alteration. However, all analyses on turbid zircon plot on the "younger side" of the chord defined by pristine domains. This suggests a multi-stage lead loss and possibility that major alteration relates to the 1.8-1.9 Ga thermal pulse (Hanski et al., 2001). 
Table 1. Ion microprobe U-Th-Pb analytical data for zircons from 2.2 Ga mafic intrusions.

\begin{tabular}{|c|c|c|c|c|c|c|c|c|c|c|c|c|c|c|c|c|}
\hline $\begin{array}{l}\text { Sample/ } \\
\text { spot\# }\end{array}$ & $\begin{array}{l}{[U]} \\
\mathrm{ppm}\end{array}$ & $\begin{array}{l}{[\mathrm{Pb}]} \\
\mathrm{ppm}\end{array}$ & $\begin{array}{l}\text { Th/U } \\
\text { meas. }\end{array}$ & $f_{206} \%$ & ${ }^{207} \mathrm{~Pb} /{ }^{235} \mathrm{U}$ & $\begin{array}{l} \pm \sigma \\
\%\end{array}$ & ${ }^{206} \mathrm{~Pb} /{ }^{238} \mathrm{U}$ & $\begin{array}{l} \pm \sigma \\
\%\end{array}$ & rho & $\begin{array}{l}\text { Disc. } \% \\
2 \sigma \text { limit }\end{array}$ & $\begin{array}{l}{ }^{207} \mathrm{~Pb} /{ }^{206} \mathrm{~Pb} \\
\text { age (Ma) }\end{array}$ & $\pm \sigma$ & $\begin{array}{l}{ }^{207} \mathrm{~Pb} /{ }^{235} \mathrm{U} \\
\text { age }(\mathrm{Ma})\end{array}$ & $\pm \sigma$ & $\begin{array}{l}{ }^{206} \mathrm{~Pb} /{ }^{238} \mathrm{U} \\
\text { age (Ma) }\end{array}$ & $\pm \sigma$ \\
\hline \multicolumn{17}{|c|}{ A892, Haaskalehto, gabbro } \\
\hline $\begin{array}{l}\text { n651-01a } \\
\text { n651-02a } \\
\text { n651-03a } \\
\text { n651-04a }\end{array}$ & $\begin{array}{l}574 \\
533 \\
724 \\
693\end{array}$ & \begin{tabular}{|l|}
334 \\
265 \\
364 \\
451
\end{tabular} & $\begin{array}{l}0.88 \\
0.14 \\
0.23 \\
1.57\end{array}$ & $\begin{array}{l}0.02 \\
* 0.01 \\
* 0.01 \\
0.03\end{array}$ & $\begin{array}{l}8.073 \\
8.080 \\
7.984 \\
8.026\end{array}$ & $\begin{array}{l}1.9 \\
1.9 \\
1.9 \\
1.9\end{array}$ & $\begin{array}{l}0.4208 \\
0.4234 \\
0.4184 \\
0.4198\end{array}$ & $\begin{array}{l}1.9 \\
1.9 \\
1.9 \\
1.9\end{array}$ & $\begin{array}{l}0.98 \\
0.99 \\
0.99 \\
0.98\end{array}$ & $\begin{array}{l}* * \\
* * \\
* * \\
* *\end{array}$ & $\begin{array}{l}2216 \\
2207 \\
2207 \\
2211\end{array}$ & $\begin{array}{l}6 \\
5 \\
4 \\
6\end{array}$ & $\begin{array}{l}2239 \\
2240 \\
2229 \\
2234\end{array}$ & $\begin{array}{l}17 \\
17 \\
17 \\
17\end{array}$ & $\begin{array}{l}2264 \\
2276 \\
2253 \\
2260\end{array}$ & $\begin{array}{l}36 \\
36 \\
36 \\
36\end{array}$ \\
\hline \multicolumn{17}{|c|}{ A865, Susivaara, metagabbro } \\
\hline $\begin{array}{l}\text { n654-01a } \\
\text { n654-02a } \\
\text { n654-02b } \\
\text { n654-03a } \\
\text { n654-03b } \\
\text { n654-04a } \\
\text { n654-05a } \\
\text { n654-06a } \\
\text { n654-07a } \\
\text { n654-07b } \\
\text { n654-08a }\end{array}$ & $\begin{array}{r}2547 \\
1858 \\
1498 \\
793 \\
1087 \\
608 \\
1062 \\
1282 \\
1070 \\
455 \\
617\end{array}$ & $\begin{array}{r}1097 \\
1282 \\
654 \\
470 \\
479 \\
280 \\
289 \\
665 \\
650 \\
225 \\
230\end{array}$ & \begin{tabular}{|l|}
0.07 \\
2.51 \\
11.34 \\
2.27 \\
5.65 \\
1.45 \\
1.05 \\
2.16 \\
2.78 \\
2.62 \\
4.75
\end{tabular} & $\begin{array}{l}0.05 \\
0.01 \\
1.23 \\
0.18 \\
0.88 \\
0.78 \\
2.67 \\
0.14 \\
0.07 \\
0.55 \\
1.64\end{array}$ & $\begin{array}{l}7.031 \\
7.112 \\
1.725 \\
6.296 \\
3.100 \\
5.162 \\
3.191 \\
5.566 \\
6.052 \\
4.790 \\
2.340\end{array}$ & $\begin{array}{l}1.1 \\
1.2 \\
2.0 \\
1.2 \\
1.3 \\
1.0 \\
1.2 \\
1.3 \\
1.2 \\
1.2 \\
1.8\end{array}$ & $\begin{array}{l}0.3721 \\
0.3760 \\
0.1231 \\
0.3312 \\
0.1976 \\
0.2999 \\
0.1911 \\
0.2978 \\
0.3233 \\
0.2761 \\
0.1540\end{array}$ & $\begin{array}{l}1.1 \\
1.2 \\
1.6 \\
1.2 \\
1.2 \\
0.9 \\
0.9 \\
1.3 \\
1.1 \\
1.1 \\
1.2\end{array}$ & $\begin{array}{l}0.98 \\
0.99 \\
0.81 \\
0.96 \\
0.88 \\
0.88 \\
0.77 \\
0.98 \\
0.98 \\
0.92 \\
0.71\end{array}$ & $\begin{array}{l}-5.9 \\
-4.8 \\
-51.7 \\
-16.2 \\
-37.7 \\
-16.3 \\
-43.0 \\
-23.4 \\
-17.3 \\
-23.2 \\
-46.1\end{array}$ & $\begin{array}{l}2190 \\
2192 \\
1655 \\
2201 \\
1860 \\
2026 \\
1973 \\
2171 \\
2174 \\
2040 \\
1803\end{array}$ & $\begin{array}{c}4 \\
3 \\
21 \\
6 \\
11 \\
8 \\
13 \\
4 \\
4 \\
9 \\
23\end{array}$ & $\begin{array}{l}2115 \\
2126 \\
1018 \\
2018 \\
1433 \\
1846 \\
1455 \\
1911 \\
1983 \\
1783 \\
1225\end{array}$ & $\begin{array}{r}10 \\
11 \\
13 \\
11 \\
10 \\
8 \\
9 \\
12 \\
10 \\
10 \\
13\end{array}$ & $\begin{array}{r}2039 \\
2058 \\
748 \\
1844 \\
1163 \\
1691 \\
1127 \\
1681 \\
1806 \\
1572 \\
923\end{array}$ & $\begin{array}{r}18 \\
21 \\
11 \\
19 \\
12 \\
13 \\
9 \\
19 \\
18 \\
16 \\
11\end{array}$ \\
\hline \multicolumn{17}{|c|}{ A1096, Koli, granophyre } \\
\hline $\begin{array}{l}\text { n655-01a } \\
\text { n655-02a } \\
\text { n655-03a } \\
\text { n655-03b } \\
\text { n655-04a }\end{array}$ & $\begin{array}{r}125 \\
1841 \\
735 \\
723 \\
1373\end{array}$ & $\begin{array}{r}72 \\
1307 \\
395 \\
360 \\
504\end{array}$ & $\begin{array}{l}3.58 \\
2.03 \\
0.99 \\
1.39 \\
6.48\end{array}$ & $\begin{array}{l}0.59 \\
0.18 \\
* 0.01 \\
0.16 \\
0.8\end{array}$ & $\begin{array}{l}4.734 \\
7.310 \\
7.142 \\
6.198 \\
2.341\end{array}$ & $\begin{array}{l}2.0 \\
2.3 \\
1.1 \\
1.2 \\
1.0\end{array}$ & $\begin{array}{l}0.3051 \\
0.3928 \\
0.3788 \\
0.3376 \\
0.1628\end{array}$ & $\begin{array}{l}1.6 \\
2.3 \\
1.1 \\
1.1 \\
0.8\end{array}$ & $\begin{array}{l}0.81 \\
0.98 \\
0.96 \\
0.95 \\
0.78\end{array}$ & $\begin{array}{c}-1.5 \\
* * \\
-3.7 \\
-11.7 \\
-42.8\end{array}$ & $\begin{array}{l}1841 \\
2164 \\
2186 \\
2140 \\
1703\end{array}$ & $\begin{array}{c}21 \\
9 \\
6 \\
7 \\
12\end{array}$ & $\begin{array}{l}1773 \\
2150 \\
2129 \\
2004 \\
1225\end{array}$ & $\begin{array}{r}17 \\
21 \\
10 \\
11 \\
7\end{array}$ & $\begin{array}{r}1716 \\
2136 \\
2071 \\
1875 \\
972\end{array}$ & $\begin{array}{r}25 \\
42 \\
19 \\
18 \\
7\end{array}$ \\
\hline \multicolumn{17}{|c|}{ A1182, Koli, gabbro } \\
\hline $\begin{array}{l}\text { n656-01a } \\
\text { n656-01b } \\
\text { n656-02a } \\
\text { n656-03a } \\
\text { n656-03b } \\
\text { n656-04a }\end{array}$ & $\begin{array}{r}364 \\
250 \\
1330 \\
638 \\
834 \\
2139\end{array}$ & \begin{tabular}{|r|}
245 \\
79 \\
156 \\
396 \\
507 \\
329
\end{tabular} & $\begin{array}{l}2.68 \\
1.60 \\
2.79 \\
1.87 \\
1.89 \\
3.89\end{array}$ & $\begin{array}{l}0.97 \\
7.9 \\
2.73 \\
0.02 \\
0.02 \\
0.78\end{array}$ & $\begin{array}{l}6.651 \\
2.895 \\
1.166 \\
7.029 \\
6.900 \\
1.289\end{array}$ & $\begin{array}{l}1.4 \\
3.3 \\
2.5 \\
2.0 \\
1.7 \\
5.0\end{array}$ & $\begin{array}{l}0.3491 \\
0.1972 \\
0.0683 \\
0.3674 \\
0.3588 \\
0.0928\end{array}$ & $\begin{array}{l}1.3 \\
1.3 \\
0.7 \\
1.8 \\
1.6 \\
4.9\end{array}$ & $\begin{array}{l}0.92 \\
0.41 \\
0.29 \\
0.90 \\
0.93 \\
0.98\end{array}$ & $\begin{array}{l}-11.3 \\
-22.0 \\
-69.2 \\
-5.3 \\
-8.9 \\
-61.2\end{array}$ & & $\begin{array}{l}9 \\
54 \\
41 \\
15 \\
11 \\
20\end{array}$ & $\begin{array}{r}2066 \\
1381 \\
785 \\
2115 \\
2099 \\
841\end{array}$ & $\begin{array}{l}12 \\
25 \\
14 \\
18 \\
15 \\
29\end{array}$ & $\begin{array}{r}1930 \\
1160 \\
426 \\
2017 \\
1977 \\
572\end{array}$ & $\begin{array}{r}21 \\
14 \\
3 \\
31 \\
27 \\
27\end{array}$ \\
\hline \multicolumn{17}{|c|}{ A977, Rahasmäki, metagabbro } \\
\hline $\begin{array}{l}\text { n653-01a } \\
\text { n653-02a } \\
\text { n653-03a } \\
\text { n653-04a } \\
\text { n653-05a } \\
\text { n653-05b } \\
\text { n653-06a } \\
\text { n653-07a } \\
\text { n653-08a } \\
\text { n653-08b } \\
\text { n653-09a } \\
\text { n653-10a }\end{array}$ & $\begin{array}{r}764 \\
429 \\
2737 \\
3038 \\
462 \\
371 \\
649 \\
570 \\
567 \\
607 \\
1899 \\
3642\end{array}$ & \begin{tabular}{|r}
438 \\
152 \\
2073 \\
486 \\
405 \\
359 \\
460 \\
396 \\
670 \\
510 \\
2062 \\
2595
\end{tabular} & \begin{tabular}{|c|}
0.68 \\
0.21 \\
13.38 \\
1.04 \\
4.67 \\
3.95 \\
4.39 \\
3.86 \\
6.63 \\
7.21 \\
31.51 \\
8.85
\end{tabular} & $\begin{array}{l}0.14 \\
0.58 \\
0.23 \\
0.62 \\
0.51 \\
0.87 \\
5.3 \\
0.56 \\
0.12 \\
0.51 \\
8.16 \\
4.13\end{array}$ & $\begin{array}{l}8.118 \\
4.588 \\
2.750 \\
1.364 \\
5.545 \\
6.430 \\
4.575 \\
5.040 \\
7.422 \\
4.299 \\
2.286 \\
3.571\end{array}$ & $\begin{array}{r}1.4 \\
1.5 \\
3.9 \\
1.1 \\
1.3 \\
2.4 \\
3.0 \\
1.4 \\
1.2 \\
1.5 \\
14.7 \\
6.6\end{array}$ & $\begin{array}{l}0.4298 \\
0.3017 \\
0.2729 \\
0.1130 \\
0.3406 \\
0.3899 \\
0.2995 \\
0.3102 \\
0.3974 \\
0.2660 \\
0.2365 \\
0.3068\end{array}$ & $\begin{array}{l}1.3 \\
1.4 \\
3.8 \\
0.9 \\
1.2 \\
2.3 \\
1.3 \\
1.3 \\
1.2 \\
1.3 \\
3.4 \\
3.0\end{array}$ & $\begin{array}{l}0.97 \\
0.93 \\
0.99 \\
0.78 \\
0.89 \\
0.97 \\
0.43 \\
0.89 \\
0.97 \\
0.85 \\
0.23 \\
0.45\end{array}$ & $\begin{array}{c}3.1 \\
-2.9 \\
46.5 \\
-48.2 \\
* * \\
4.5 \\
* * \\
-7.1 \\
* * \\
-19.0 \\
* * \\
0.3\end{array}$ & $\begin{array}{r}2190 \\
1804 \\
1016 \\
1372 \\
1927 \\
1950 \\
1812 \\
1924 \\
2170 \\
1914 \\
931 \\
1302\end{array}$ & $\begin{array}{c}5 \\
10 \\
13 \\
14 \\
10 \\
11 \\
49 \\
12 \\
5 \\
14 \\
268 \\
111\end{array}$ & $\begin{array}{r}2244 \\
1747 \\
1342 \\
873 \\
1908 \\
2036 \\
1745 \\
1826 \\
2164 \\
1693 \\
1208 \\
1543\end{array}$ & $\begin{array}{r}13 \\
13 \\
29 \\
7 \\
11 \\
21 \\
26 \\
12 \\
11 \\
12 \\
109 \\
54\end{array}$ & $\begin{array}{r}2305 \\
1700 \\
1555 \\
690 \\
1890 \\
2122 \\
1689 \\
1742 \\
2157 \\
1521 \\
1368 \\
1725\end{array}$ & $\begin{array}{r}26 \\
21 \\
53 \\
6 \\
19 \\
42 \\
20 \\
19 \\
21 \\
17 \\
42 \\
46\end{array}$ \\
\hline \multicolumn{17}{|c|}{ A586, Ensilä, metagabbro } \\
\hline $\begin{array}{l}\text { n657-01a } \\
\text { n657-02a } \\
\text { n657-03a } \\
\text { n657-04a } \\
\text { n657-05a } \\
\text { n657-05b }\end{array}$ & $\begin{array}{r}560 \\
777 \\
382 \\
1381 \\
900 \\
455\end{array}$ & \begin{tabular}{|l}
217 \\
354 \\
264 \\
825 \\
666 \\
252
\end{tabular} & \begin{tabular}{|l|}
0.33 \\
11.38 \\
2.72 \\
2.10 \\
7.87 \\
3.12
\end{tabular} & $\begin{array}{l}{ }^{*} 0.01 \\
4.22 \\
0.08 \\
0.02 \\
6.68 \\
2.48\end{array}$ & $\begin{array}{l}5.060 \\
4.821 \\
6.930 \\
6.605 \\
4.494 \\
4.755\end{array}$ & $\begin{array}{l}1.4 \\
1.6 \\
1.9 \\
1.2 \\
7.4 \\
3.1\end{array}$ & $\begin{array}{l}0.3197 \\
0.2599 \\
0.3615 \\
0.3468 \\
0.2496 \\
0.2597\end{array}$ & $\begin{array}{l}1.3 \\
1.1 \\
1.9 \\
1.2 \\
0.8 \\
2.6\end{array}$ & $\begin{array}{l}0.96 \\
0.69 \\
0.97 \\
0.98 \\
0.11 \\
0.85\end{array}$ & $\begin{array}{c}-2.2 \\
-29.4 \\
-8.0 \\
-12.7 \\
-4.5 \\
-26.2\end{array}$ & $\begin{array}{l}1876 \\
2158 \\
2215 \\
2204 \\
2106 \\
2135\end{array}$ & $\begin{array}{c}7 \\
21 \\
8 \\
4 \\
123 \\
28\end{array}$ & $\begin{array}{l}1829 \\
1789 \\
2103 \\
2060 \\
1730 \\
1777\end{array}$ & $\begin{array}{l}12 \\
14 \\
17 \\
11 \\
63 \\
26\end{array}$ & $\begin{array}{l}1788 \\
1489 \\
1989 \\
1919 \\
1437 \\
1488\end{array}$ & $\begin{array}{l}21 \\
15 \\
32 \\
20 \\
11 \\
35\end{array}$ \\
\hline
\end{tabular}

$\mathrm{f}_{206} \%$ : Percentage of ${ }^{206} \mathrm{~Pb}$ contributed by common $\mathrm{Pb}$, assuming $\mathrm{Pb}$ isotopic composition at 1.9 Ga (Stacey and Kramers, 1975).

$\mathrm{f}_{206} \%$ : *0.01 - insignificant amount of common $\mathrm{Pb}$.

Disc. \%: Discordance of data (if $>2 \sigma$ error of analysis). ${ }^{* *}$ indicates that analysis is concordant within $2 \sigma$ error.

Sample coordinates: A892: $X=7488.49, Y=3464.70$; A865: $X=7345.14, Y=2513.90 ; A 586: X=7132.99, Y=4456.79 ; A 977: X=7020.02$, $\mathrm{Y}=3548.98$

A1096: $\mathrm{X}=6991.79, \mathrm{Y}=$ 4498.06; $\mathrm{A} 1182: \mathrm{X}=6992.35, \mathrm{Y}=4497.80$

Coordinates are presented in the Finnish kkj-coordinate system. 


\section{A865 Susivaara}
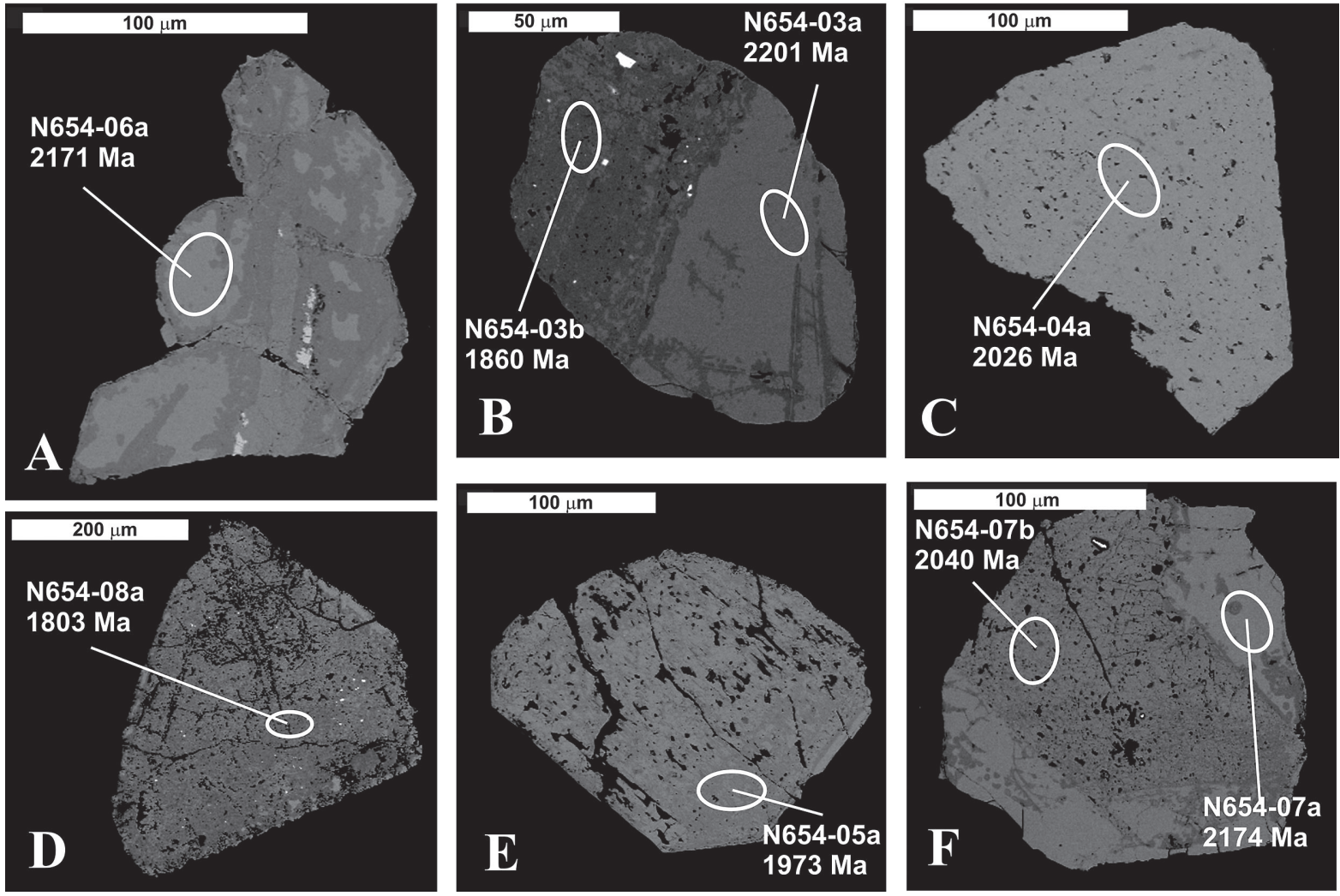

Fig. 4. BSE images of zircon grains from the Susivaara sill (sample A865). All annotated ages are ${ }^{207} \mathrm{~Pb} /{ }^{206} \mathrm{~Pb}$ ages, which may differ from upper intercept ages depending on degree of discordance.

\section{A865 Susivaara}

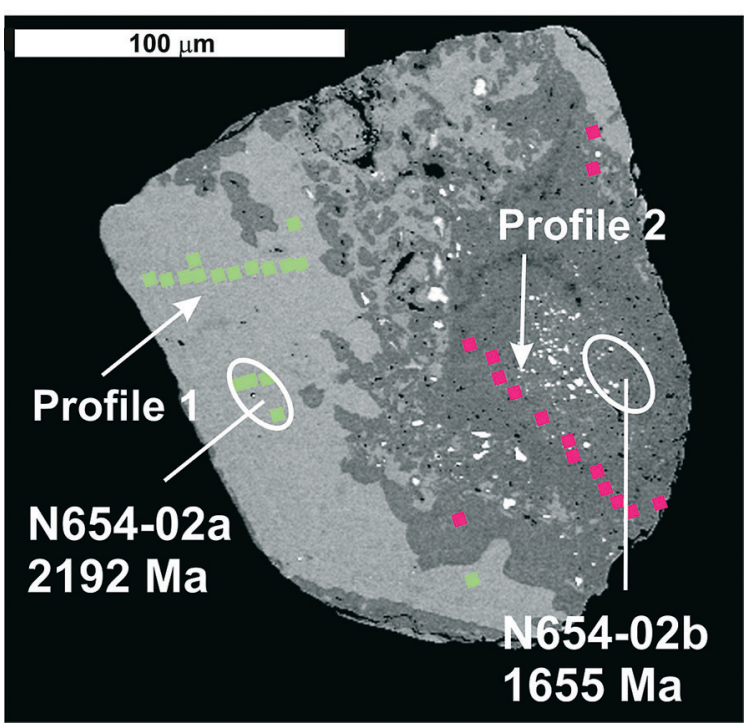

Fig. 5. Spots of electron microprobe analyses (see Table 3 ) with red and green symbols representing "altered" and "fresh" domains, respectively.

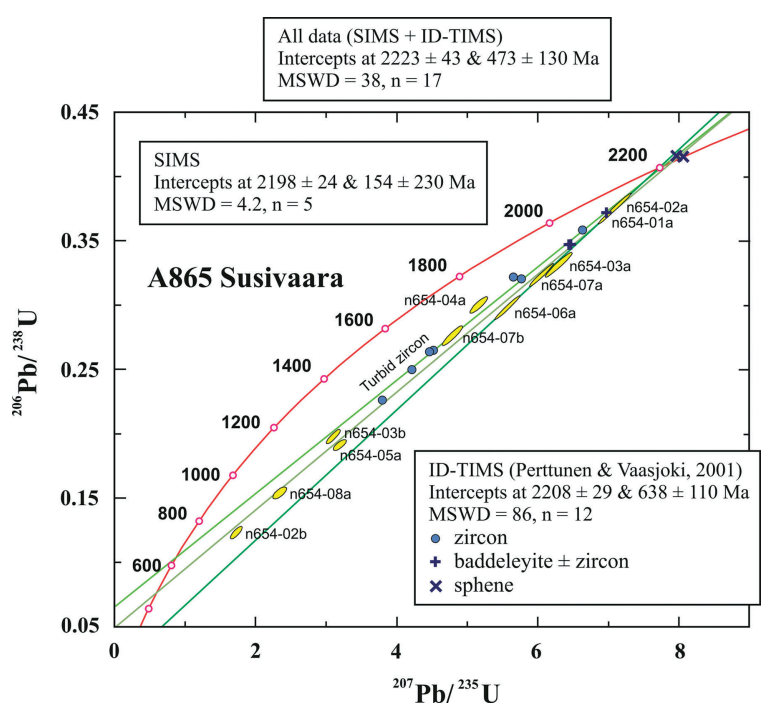

Fig. 6. Concordia plots of U-Pb data of sample A865 from the Susivaara intrusion, Peräpohja Schist Belt. SIMS analyses shown as yellow error ellipsoids and ID-TIMS analyses by blue symbols without error indication. 


\subsubsection{Ensilä (A586)}

Most zircon grains in sample A586 from the Ensilä metagabbro are turbid, but BSE images reveal the existence of grains that are less altered or even very clean (Fig. 7). Magmatic zoning is still present in some grains (Fig. 7C). In Fig. $7 \mathrm{~F}$ is shown the BSE image of an interesting grain having three different types of material: a broad, light-colored, fractured rim, representing the most pristine zircon, encloses a non-fractured and obviously more altered interior, which is composed of a complicated patchwork of light-colored and dark-colored areas. The central part of this grain shows that even though a BSE image reveals two or more contrasting shades in terms of BSE intensity, none of them necessarily represents unaltered zircon.

Five conventional multi-grain U-Pb analyses indicate rather high contents of common lead for zircon in sample A586. The isotopic data are discordant and heterogeneous providing ${ }^{207} \mathrm{~Pb} /{ }^{206} \mathrm{~Pb}$ ages from 1.95 to $2.12 \mathrm{Ga}$ (Table 2, Fig.8). Also shown in the diagram are $\mathrm{U}-\mathrm{Pb}$ zircon data reported by Hyppönen (1983) for two gabbroic samples of the same rock association, 12 and $15 \mathrm{~km}$ south of our site, respectively (A491 from Hietaperä and A910 from Petäjäniemi). These data are similarly heterogeneous precluding precise age calculation.

The ion microprobe was used for six measurements from five zircon crystals, but due to a high amount of common lead, one analysis (n657-05a) has been omitted from further discussion. Two analyses (n657-03a, n657-04a) from the well-preserved and relatively clear, though unzoned zircon grains yield moderately discordant data with ${ }^{207} \mathrm{~Pb} /$ ${ }^{206} \mathrm{~Pb}$ ages of c. $2.2 \mathrm{Ga}$, whereas two analyses (n65702a, n657-05b) from more altered zircon grains are significantly discordant (Fig. 8). A discordia forced

\section{A586 Ensilä}
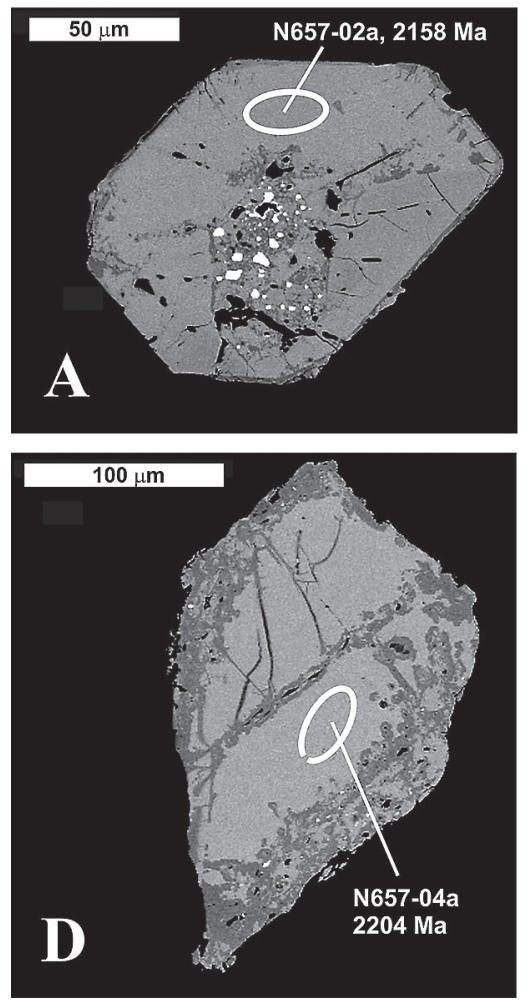
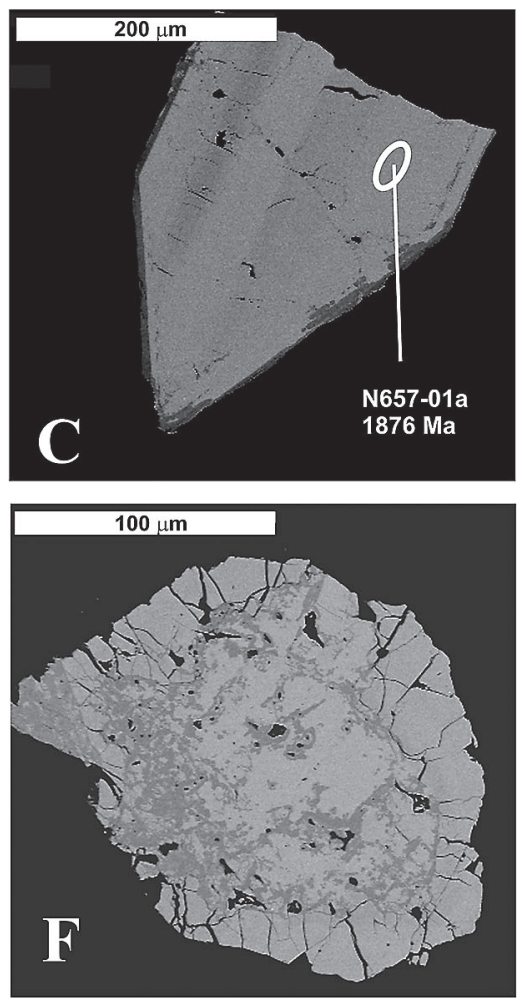

Fig. 7. BSE images of zircon grains from the Ensilä gabbro (sample A586). All annotated ages are ${ }^{207} \mathrm{~Pb} /{ }^{206} \mathrm{~Pb}$ ages, which may differ from upper intercept ages depending on degree of discordance. Note the central part of the zircon grain in figure A, containing many thorite inclusions. 


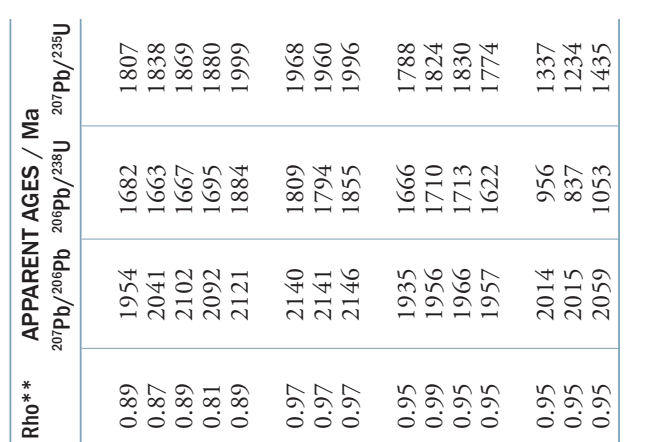

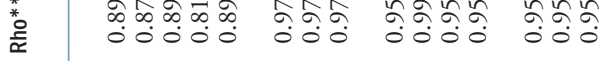

ํำ

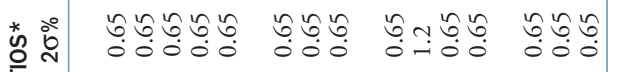

否

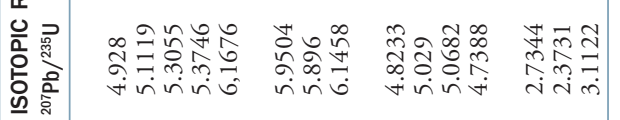

$\stackrel{\overline{\bar{\alpha}}}{\underline{0}}$

들

을

$\frac{1}{3}$

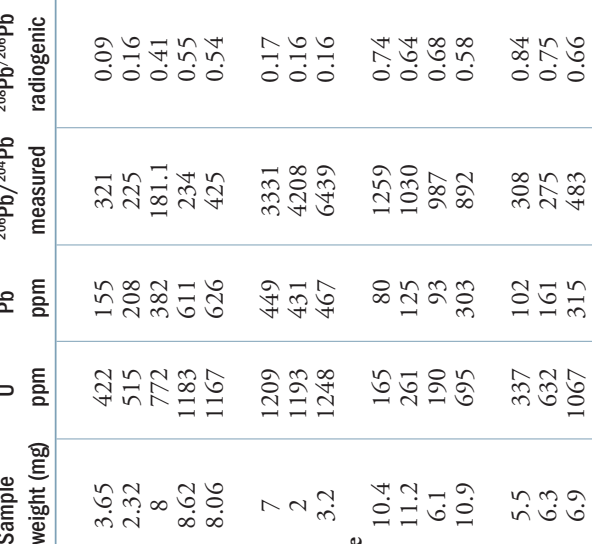

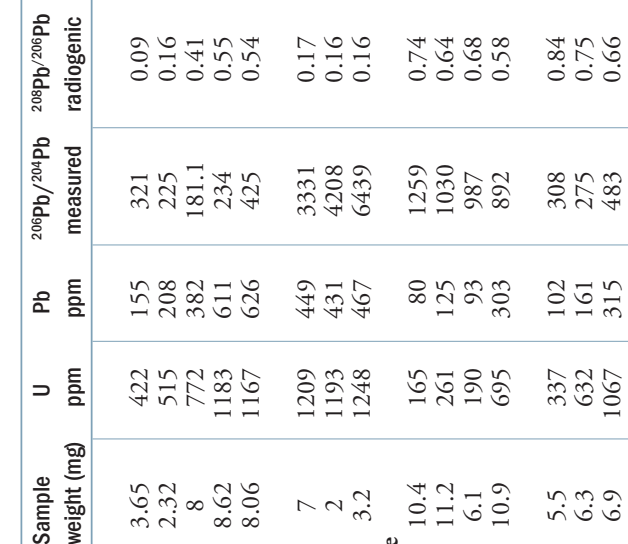

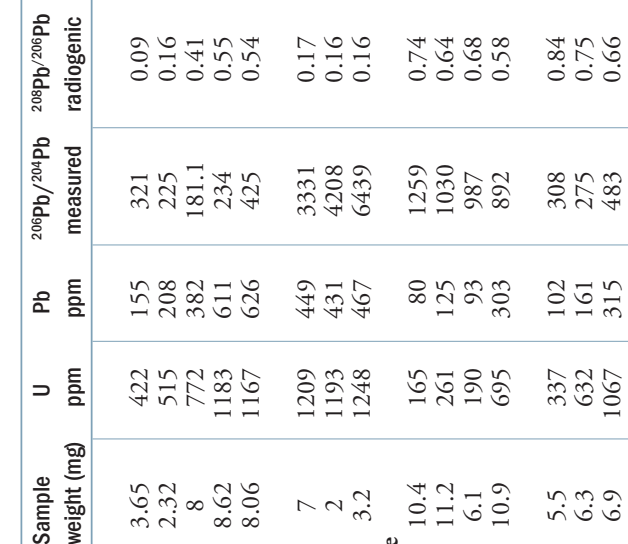

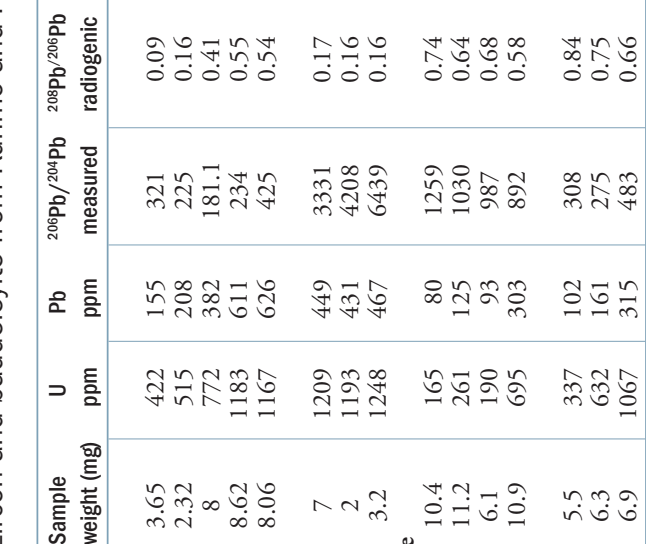

$\stackrel{\pi}{\frac{0}{0}}$

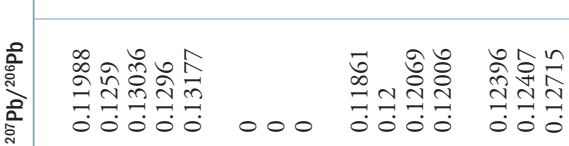

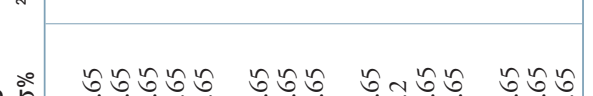

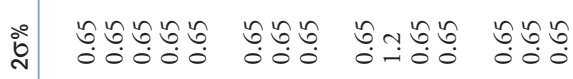

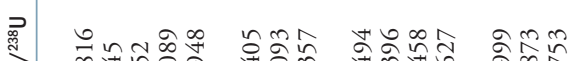

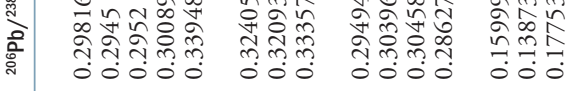

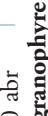
衰 范

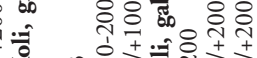

造 0

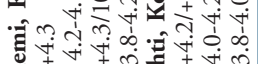
.ดัง

Fig. 8. Concordia plots of U-Pb data of three samples (A491, A586, A910) from Paleoproterozoic mafic intrusive rocks from the Archean Kuhmo Greenstone Belt. SIMS analyses shown as yellow error ellipsoids and ID-TIMS analyses by blue and green circles without error indication.

through these four analyses gives an upper intercept age of $2232 \pm 19$ Ma. Analysis n65701 a is clearly distinct from the other and provides a nearly concordant ${ }^{207} \mathrm{~Pb} /{ }^{206} \mathrm{~Pb}$ age of c. 1.9 Ga. This analysis also has a lower $\mathrm{Th} / \mathrm{U}$ ratio (0.3) compared to other data (Table 1, Fig. 8). The grain in question is relatively transparent and, surprisingly, possesses a weak zoning pattern. One possibility is that the grain is an outsider introduced by contamination during sample preparation. On the other hand, it represents the heaviest zircon fraction in the sample $\left(\mathrm{d}>4.2 \mathrm{~g} / \mathrm{cm}^{3}\right)$, and the same features are obvious from the conventional data, i.e. analysis A586A from the heaviest zircon fraction has the lowest ${ }^{207} \mathrm{~Pb} /{ }^{206} \mathrm{~Pb}$ age and $\mathrm{Th} / \mathrm{U}$ ratio (as deduced from the ${ }^{208} \mathrm{~Pb} / 206 \mathrm{~Pb}$ data in Table 2). Note that dark, low- $Z$ areas within the zircon crystals were not analyzed by SIMS from sample A586 as all the analyzed spots represent relatively high-intensity BSE areas (see Fig. 7).

In summary, most of the multi-grain zircon fractions analyzed by the conventional method plot within the area delineated by the spot analyses (Fig. 8). The new data confirm that the Ensilä intrusion belongs to the c. 2.2 
Ga age group and suggest that the intrusive body has undergone a multi-stage evolution including significant resetting of the zircon $\mathrm{U}-\mathrm{Pb}$ system at $\mathrm{c}$. 1.8-1.9 Ga. This means that the surrounding Archean greenstone belt has also experienced the same tectono-metamorphic phenomena in the $\mathrm{Pa}$ leoproterozoic time.

\subsubsection{Rahasmäki (A977)}

Zircons in the Rahasmäki intrusion are commonly very turbid but, as revealed by BSE images, some of the grains still retain well-preserved zones (Fig. 9). An interesting feature of the conventional data from Rahasmäki, as published by Paavola (1984), is that the heavier zircon grains (density $4.0-4.2 \mathrm{~g} / \mathrm{cm}^{3}$ ) have higher $\mathrm{U}$ contents and are more discordant than the lighter ones $\left(3.8-4.0 \mathrm{~g} / \mathrm{cm}^{3}\right)$. Normally the situation is reversed (see Table 2 ).
Twelve U-Pb analyses on ten grains were obtained by ion microprobe. Three analyses with high amounts of common lead $\left({ }^{206} \mathrm{~Pb} /{ }^{204} \mathrm{~Pb}<400\right)$ and consequently large errors have been omitted from the concordia diagram and further discussion. The data as a whole are very scattered (Table 1, Fig. 10). Four analyses plot close to the chord defined by the conventional data of Paavola (1984), but two analyses from the most pristine zircon domains are nearly concordant at c. $2.2 \mathrm{Ga}$ (n653-01a, n653-08a). This difference is clearly seen between the two spots in grain n653-08, which exemplifies the complicated alteration pattern seen in BSE images of many crystals (Fig. 9E). One analysis (n653-02a) is slightly on the "younger side" of the chord, but analysis n653-03a plots above the concordia with a ${ }^{207} \mathrm{~Pb} /$ ${ }^{206} \mathrm{~Pb}$ age of c. $1.0 \mathrm{Ga}$. The analysis indicates a very high Th content (c. $4 \%$ ), and it is expected that the analytical routine for normal zircon may not be

\section{A977 Rahasmäki}
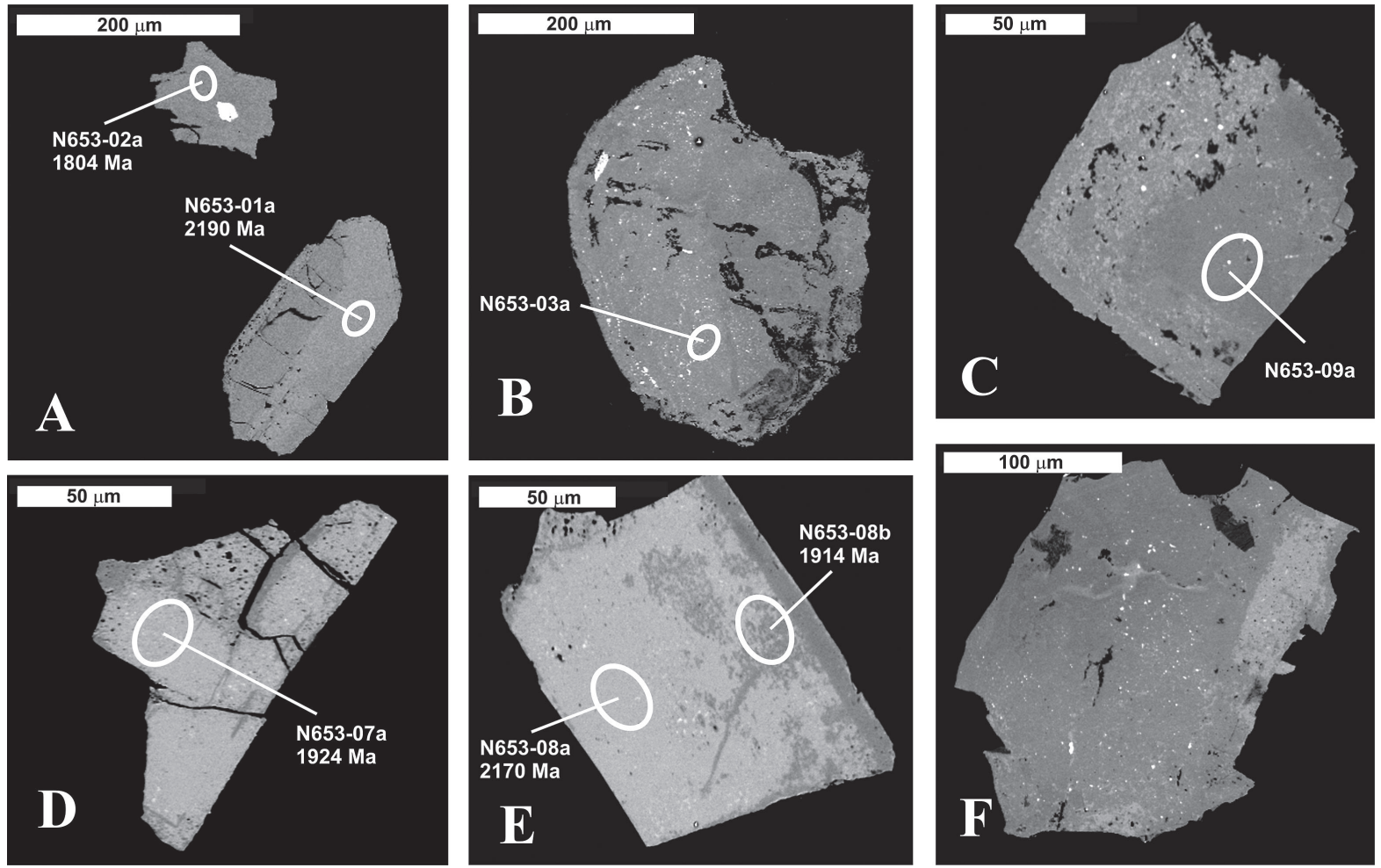

Fig. 9. BSE images of zircon grains from the Rahasmäki sill (sample A977). All annotated ages are ${ }^{207} \mathrm{~Pb} /{ }^{206} \mathrm{~Pb}$ ages, which may differ from upper intercept ages depending on degree of discordance. Note the large, white baddelyite inclusion in the smaller zircon grain in figure A. The largest inclusion in the zircon grain of figure $B$ is thorite. 


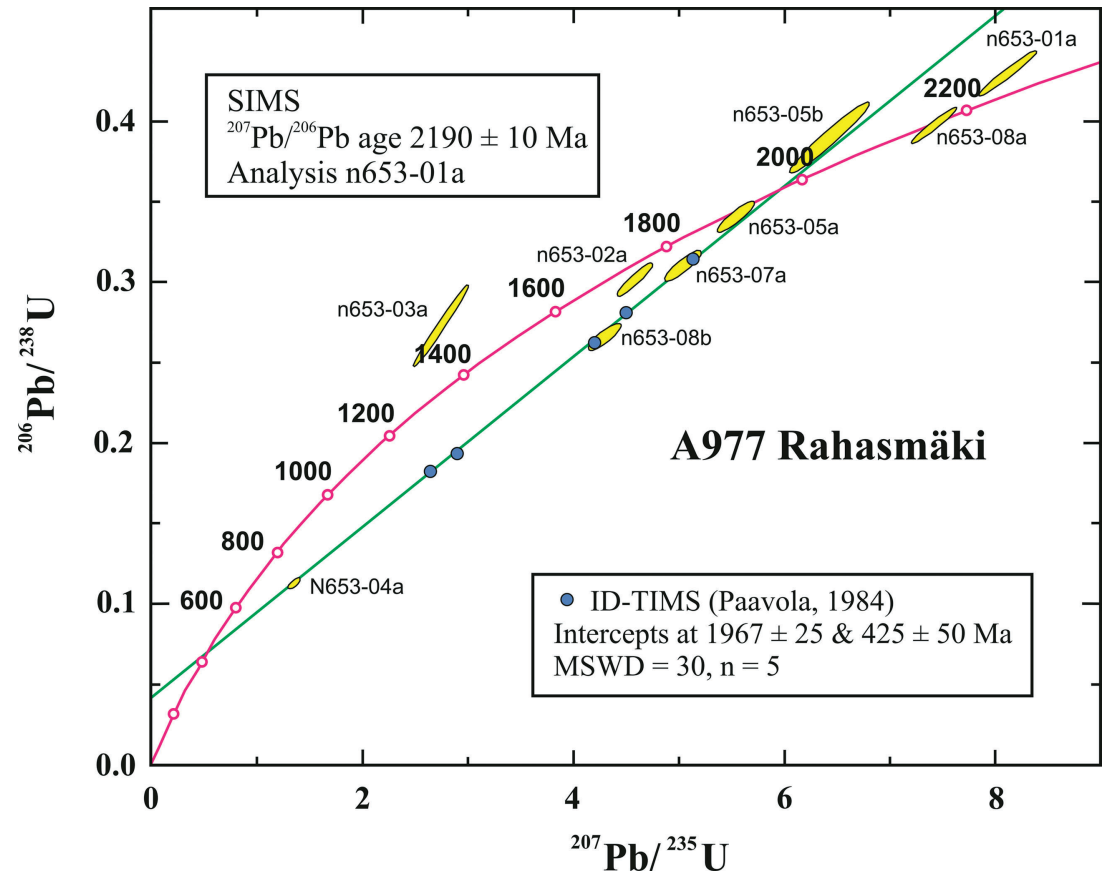

Fig. 10. Concordia plot of $\mathrm{U}-\mathrm{Pb}$ data of sample A977 from the Rahasmäki sill, TahkomäkiKinahmi schist belt. SIMS analyses shown as yellow error ellipsoids and ID-TIMS analyses by blue circles (without error indication). valid for such a composition. The white specks in the photomicrograph of grain n653-03a (Fig. 9B) are thorite grains which further highlights the complicated isotopic history of the zircon grain.

In summary, the SIMS results from the Rahasmäki sample suggest magmatic crystallization at c. 2.2 Ga, i.e. c. $200 \mathrm{Ma}$ earlier than indicated by the previous ID-TIMS data $(1967 \pm 24 \mathrm{Ma})$, and a major lead loss from zircon at c. 1.8-1.9 Ga.

\subsubsection{Koli (A1096, A1182, A587)}

Zircon in all samples is turbid. Especially in the granophyre A1096 it occurs as large, broken, very turbid grains. As shown by the BSE images of Fig. 11, zircon grains from sample A1096 are, together with the A865 zircons from Susivaara, the most altered with a particularly spongy internal texture. Zircon crystals from the gabbro sample A1182 are notably variable in terms of preservation. Some grains are almost devoid of signs of alteration (Fig. 12C), some are traversed by a distinctive alteration band (Fig. $12 \mathrm{~F})$, and there also exist grains that are heterogeneously but pervasively altered (Fig. 12B, D). In Fig. 12E, the primary magmatic zoning is still re- cognizable in a remnant of pristine zircon close to the edge of the grain. The rest of the grain is composed of an irregular network of light-colored and grey secondary zircon enclosing a great number of silicate inclusions.

Four conventional U-Pb zircon analyses from sample A1096 are discordant and plot close to each other yielding ${ }^{207} \mathrm{~Pb} /{ }^{206} \mathrm{~Pb}$ ages of c. $1.96 \mathrm{Ga}$ (Table 2, Fig. 13A). Air abrasion has no effect on the concordance of analysis, which is understandable after inspection of the BSE images (Fig. 11). Three IDTIMS analyses of baddeleyite from sample A587 are discordant with ${ }^{207} \mathrm{~Pb} /{ }^{206} \mathrm{~Pb}$ ages of c. $2.14 \mathrm{Ga}$ (Fig. 13B). A linear regression through these data gives concordia intercepts at $2170 \pm 20 \mathrm{Ma}$ and 253 $\pm 150(\mathrm{MSWD}=1.3, \mathrm{n}=3$ ). As shown in Fig. 13B, the three conventional $\mathrm{U}-\mathrm{Pb}$ analyses made on zircon from the gabbro sample A1182 are extremely discordant and plot close to the chord defined by the baddeleyite fractions.

Eleven spot analyses on zircon were performed by SIMS from samples A1096 and A1182. Two of the analyses (n656-01b and n656-02b) have large proportions of common lead and thus large errors. The data are heterogeneous and show a very large 


\section{A1096 Kaunisniemi (Koli)}
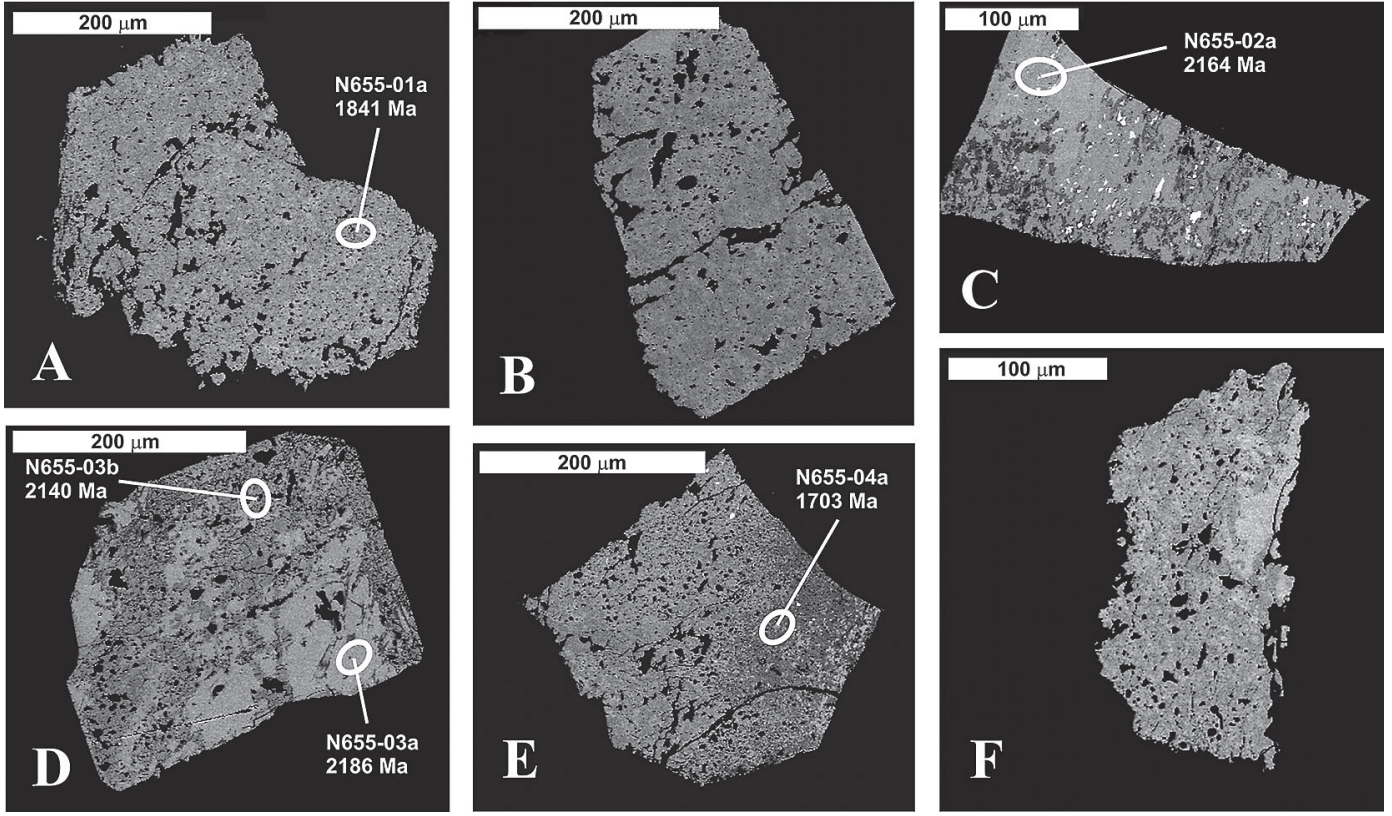

Fig. 11. BSE images of zircon grains from granophyre of the Koli sill (sample A1096 from Kaunisniemi). All annotated ages are ${ }^{207} \mathrm{~Pb} /{ }^{206} \mathrm{~Pb}$ ages, which may differ from upper intercept ages depending on degree of discordance.

\section{A1182 Kaunislahti (Koli)}
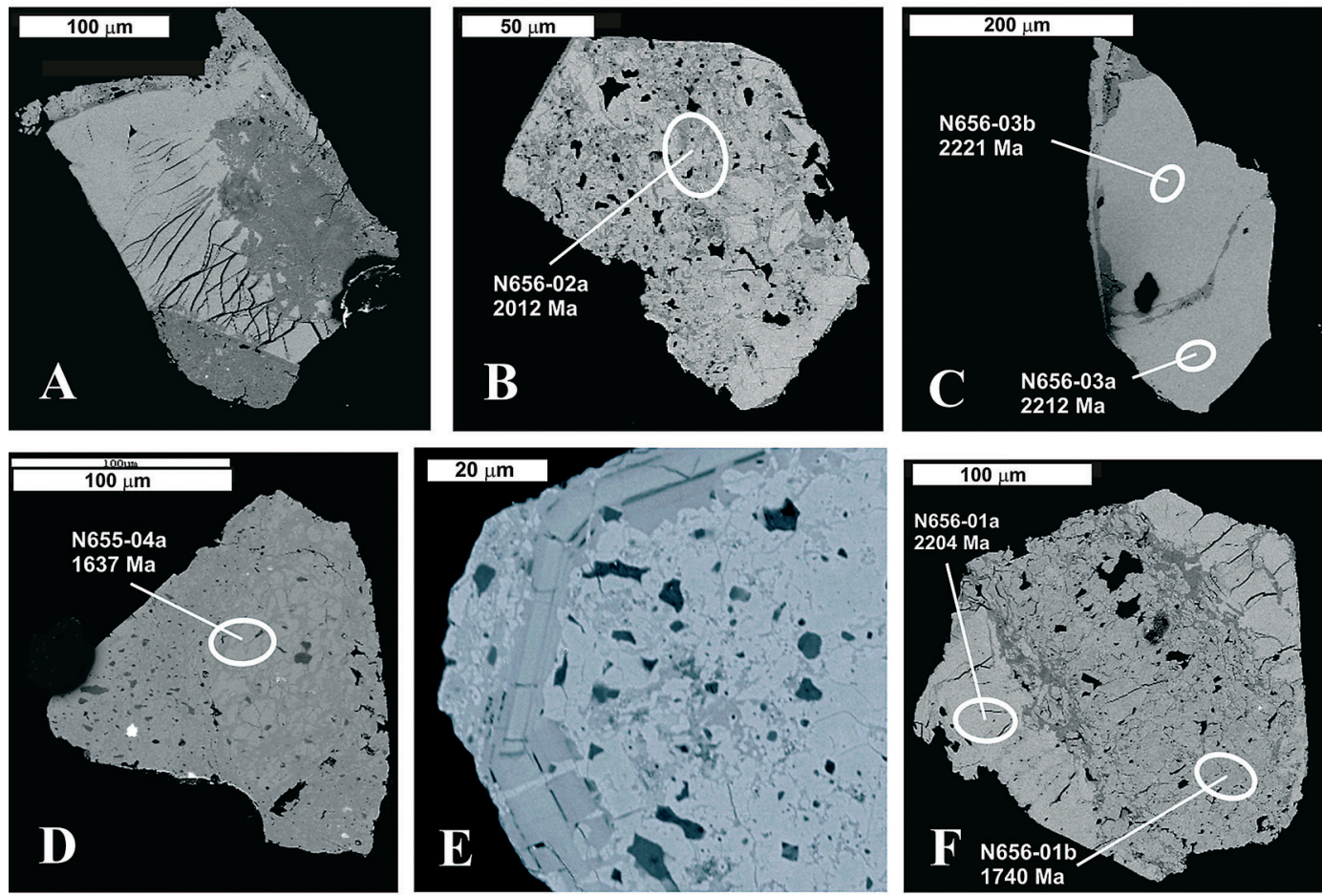

Fig. 12. BSE images of zircon grains from metagabbro of the Koli sill (sample A1182). All annotated ages are ${ }^{207} \mathrm{~Pb} /{ }^{206} \mathrm{~Pb}$ ages, which may differ from upper intercept ages depending on degree of discordance. 

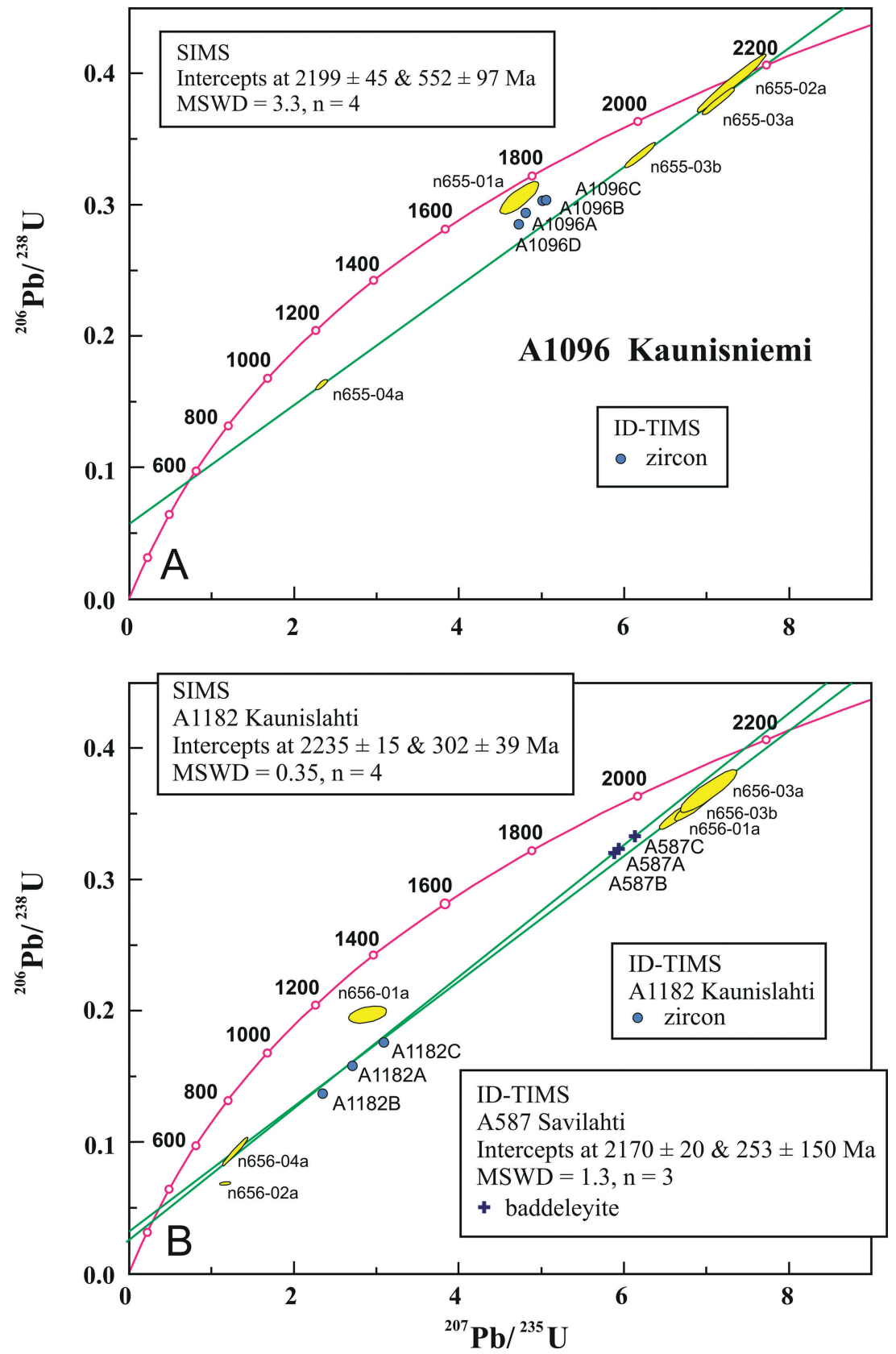

Fig. 13. Concordia plot of $\mathrm{U}-\mathrm{Pb}$ data from the Koli sill. SIMS analyses shown as yellow error ellipsoids and ID-TIMS analyses by blue circles (zircon) and crosses (baddeleyite) without error indication. A. Granophyre A1096 from Kaunisniemi. B. Gabbro A1182 from Kaunislahti and gabbro A587 from Savilahti. variation in the discordance and $U$ content. From the BSE images it is clear that the least discordant analyses (n655-02a, n655-03a, n656-03a and n65603b; Figs. 11, 12) with ${ }^{207} \mathrm{~Pb} /{ }^{206} \mathrm{~Pb}$ ages approaching 2.2 Ga represent most pristine zircon domains. Regression of four analyses from the granophyre A1096 define intercepts at $2199 \pm 45$ and $552 \pm 97$ $\mathrm{Ma}(\mathrm{MSWD}=3.3$, Fig. 13A). One SIMS analysis (n655-01a) as well as the conventional data plot distinctly on the "younger" side of the chord, suggesting significant influence of the 1.8-1.9 Ga thermal fluids in zircon. In fact, analysis n655-01a suggests complete resetting of the U-Pb system and/or formation of new zircon at c. 1.9 Ga. This grain also has a much lower $U$ content than the other grains, but surprisingly, in the BSE image (see Fig. 
$11 \mathrm{~A})$, is not very distinct from the other altered zircon crystals from the same sample.

The least discordant data from sample A1182 provide an average ${ }^{207} \mathrm{~Pb} /{ }^{206} \mathrm{~Pb}$ age of $2211 \pm 12$ $\mathrm{Ma}$, and the two very discordant points from strongly altered domains together with conventional data manifest near-complete and relatively recent loss of radiogenic lead (Fig. 13B).

The most concordant zircon analyses have a $\mathrm{U}$ content of less than 1000 ppm while discordant zircons often display a $U$ content of higher than 1000 ppm (Table 1). However, the relationship between the $\mathrm{U}$ content and degree of discordance is not simple as the above generalization does not always hold.

\subsection{Electron microprobe analyses of altered zircons}

Our electron microprobe data include 33 analyses and are restricted to a single zircon grain from sample A865 representing a gabbro from the Susivaara sill. As shown in Fig. 5, this grain exhibits a typical alteration pattern of the GWA zircons with two distinctly different shades in the BSE image, i.e. darker, patchy zones representing more altered parts and lighter, cleaner zones representing less damaged parts. In the following we refer to these two areas as altered and fresh domains, respectively. The two SIMS analyses, n654-02a and 0654-02b, were made on the same grain and resulted in clearly different ${ }^{207} \mathrm{~Pb} /{ }^{206} \mathrm{~Pb}$ ages of $2192 \pm 3$ and $1655 \pm$ $21 \mathrm{Ma}$, respectively (Table 1 ). In fact, these SIMS spots represent the two extremes in the degree of discordance of the analyses from sample A865 (Fig. 6). As indicated in Fig. 5, we analyzed two profiles by electron microprobe, one crossing a fresh zone and the other an altered zone. These were supplemented by five analyses on the SIMS crater n654$02 \mathrm{a}$ and six scattered points elsewhere in the grain. Representative analyses are shown in Table 3. In spite of the limited number of analyses, we believe that the data obtained from this single grain are representative of the main chemical characteristics of altered zircon grains in sample A865 or in the GWA rocks in general.

\begin{tabular}{|c|c|c|c|c|}
\hline & $\ddot{\sim}$ & 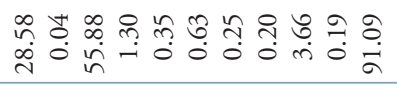 & $u$ & \\
\hline$\dot{\widehat{N}}$ & ปี & 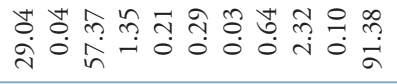 & $\cup$ & \\
\hline 崩 & $\vec{\sim}$ & 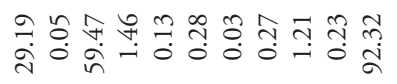 & $\cup$ & \\
\hline $\begin{array}{l}\overrightarrow{0} \\
\stackrel{\circ}{\infty} \\
+\infty\end{array}$ & ㄱ. & 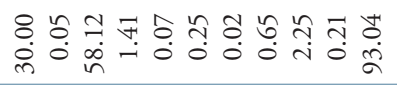 & $\cup$ & \\
\hline$\frac{\bar{d}}{\frac{1}{0}}$ & $\curvearrowright$ & 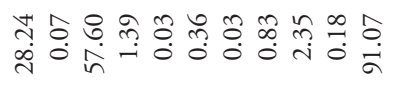 & $\cup$ & \\
\hline$\sum_{\infty}^{\infty}$ & $\stackrel{\infty}{=}$ & 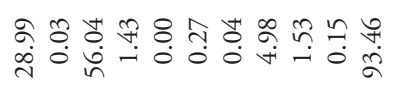 & $\cup$ & \\
\hline $\begin{array}{l}\frac{C}{0} \\
\mathscr{D}\end{array}$ & $\triangleq$ & 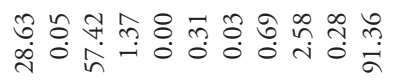 & $u$ & \\
\hline$\frac{4}{0}$ & $\stackrel{\bullet}{\sim}$ & 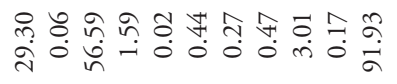 & $u$ & \\
\hline $\begin{array}{l}\stackrel{4}{0} \\
\frac{1}{0}\end{array}$ & $\cong$ & 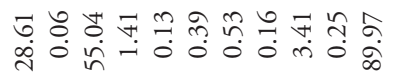 & $u$ & \\
\hline 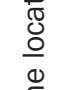 & 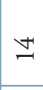 & 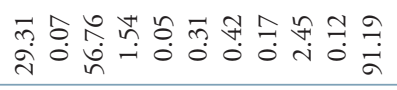 & $\cup$ & \\
\hline$\frac{1}{\grave{c}}$ & 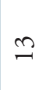 & 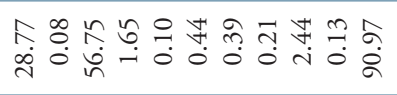 & $u$ & \\
\hline 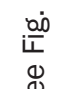 & $\cong$ & 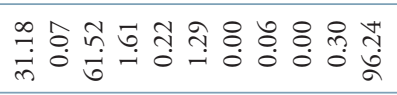 & $\infty$ & \\
\hline 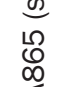 & $=$ & 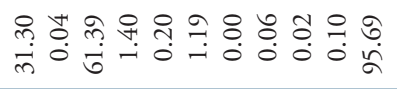 & $\infty$ & \\
\hline$\frac{0}{\frac{0}{O}}$ & $\circ$ & 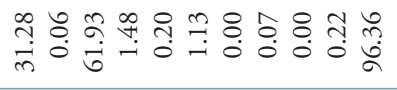 & $\infty$ & \\
\hline $\begin{array}{l}\text { थ } \\
\text { ஸे }\end{array}$ & $a$ & 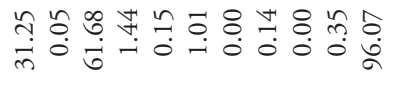 & $\infty$ & \\
\hline 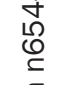 & $\infty$ & 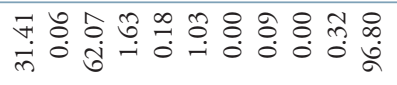 & $\infty$ & \\
\hline$\frac{5}{\frac{\pi}{20}}$ & $\Lambda$ & 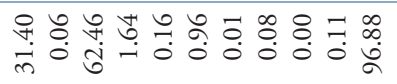 & $\infty$ & \\
\hline .0 & $\bullet$ & 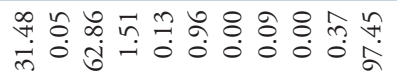 & $\infty$ & 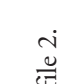 \\
\hline 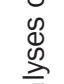 & $n$ & 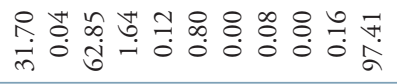 & $\infty$ & $\begin{array}{l}\stackrel{0}{a} \\
\text { ü } \\
u\end{array}$ \\
\hline $\begin{array}{l}\frac{\sigma}{c} \\
\frac{1}{\sigma} \\
0\end{array}$ & 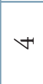 & 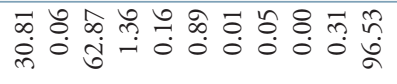 & $\varangle$ & $\stackrel{\ddot{\vec{u}}}{\frac{\tilde{I}}{\mathrm{~F}}}$ \\
\hline $\begin{array}{l}\text { 응 } \\
\text { 인 }\end{array}$ & $m$ & 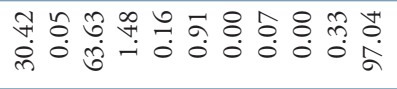 & $\varangle$ & $\stackrel{\vec{a}}{\| \prime}$ \\
\hline $\begin{array}{l}\text { है } \\
\text { 은 }\end{array}$ & N & 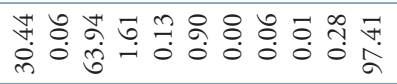 & $\varangle$ & 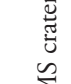 \\
\hline$\frac{\bar{d}}{\frac{\tilde{d}}{\Psi}}$ & - & 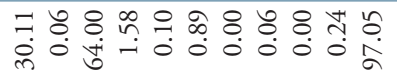 & $\varangle$ & 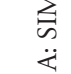 \\
\hline $\begin{array}{l}\dot{m} \\
\frac{0}{0} \\
\frac{0}{\sigma}\end{array}$ & & 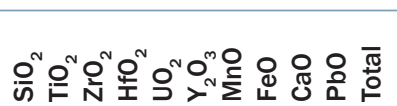 & $\begin{array}{l}\text { 응 } \\
\text { ్ㅠㅇ }\end{array}$ & 苛 \\
\hline
\end{tabular}


Figure 14 displays the analytical data divided into two groups that represent the "fresh" and "altered" domains. One of the most striking features of the altered domains is their elevated $\mathrm{CaO}$ content, which can be used as a simple criterion for distinction between relatively fresh and altered parts of zircon (cf. Geisler \& Schleicher, 2000). In the former domains, the $\mathrm{CaO}$ content is always very low $(\leq 0.02$ wt.\%), approaching or being below the detection limit of the electron microprobe, whereas in the altered domains, $\mathrm{CaO}$ is commonly higher than 1.0 wt. $\%$ and reaches a maximum value of $3.6 \mathrm{wt} . \%$ (Fig. 14B).

A characteristic feature of the altered domains is the low analytical total varying from 90.0 to 93.5 wt.\%. It is likely that the low totals are mostly accounted for by the presence of a water species in the damaged parts of the zircons (cf. Geisler et al., 2003a). Because the $\mathrm{Al}_{2} \mathrm{O}_{3}$ content was not analyzed by microprobe, we cannot know the exact amount of $\mathrm{H}_{2} \mathrm{O}$ in zircons. However, later SEMEDS analyses of altered zircons in other samples indicate approximate $\mathrm{Al}_{2} \mathrm{O}_{3}$ contents in the range of 1-2.5 wt.\%, which suggest that the water concentrations in altered domains can reach many percentages. The $\mathrm{SiO}_{2}$ and $\mathrm{ZrO}_{2}$ contents are lower in the altered domains than in the fresh domains (Fig. 14A) and can be explained by a high degree of hydration. The atomic $\mathrm{Si} /(\mathrm{Zr}+\mathrm{Hf})$ ratio in both zones overlaps and approaches stoichiometric 1.0, though on average it is slightly higher in the altered domains (0.99-1.07) compared with the fresh domains (0.95-1.03).

The $\mathrm{FeO}$ content in fresh domains is commonly less than 0.15 wt. $\%$ while in altered domains it is higher and, excluding two analyses (ca. 6 wt.\%), falls in the range of $0.15-1.0$ wt.\% (Fig. 14C). The $\mathrm{MnO}$ contents are negligible in fresh zones and also low $(<0.1 \mathrm{wt} . \%)$ in some analyses from altered zones but rises to values between 0.25 and $0.53 \mathrm{wt} . \%$ in some other parts of the altered zones (Fig. 14D). The $\mathrm{Y}_{2} \mathrm{O}_{3}$ content of the altered domains is commonly less than that in the fresh domains with the averages being $0.37 \mathrm{wt} . \%$ and $0.96 \mathrm{wt} . \%$, respecti-
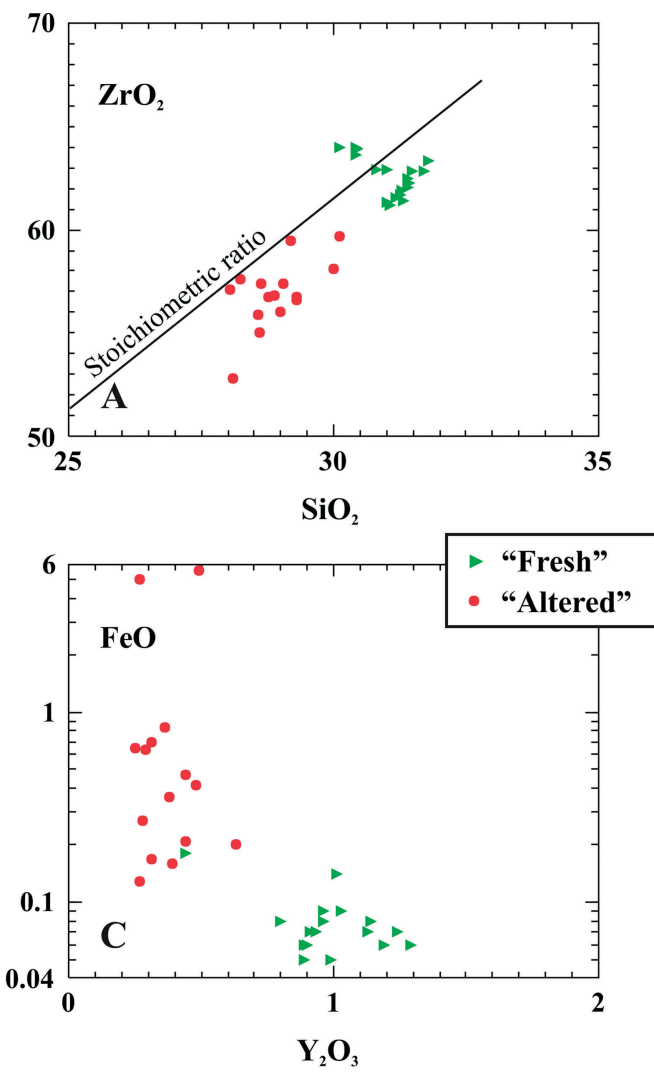
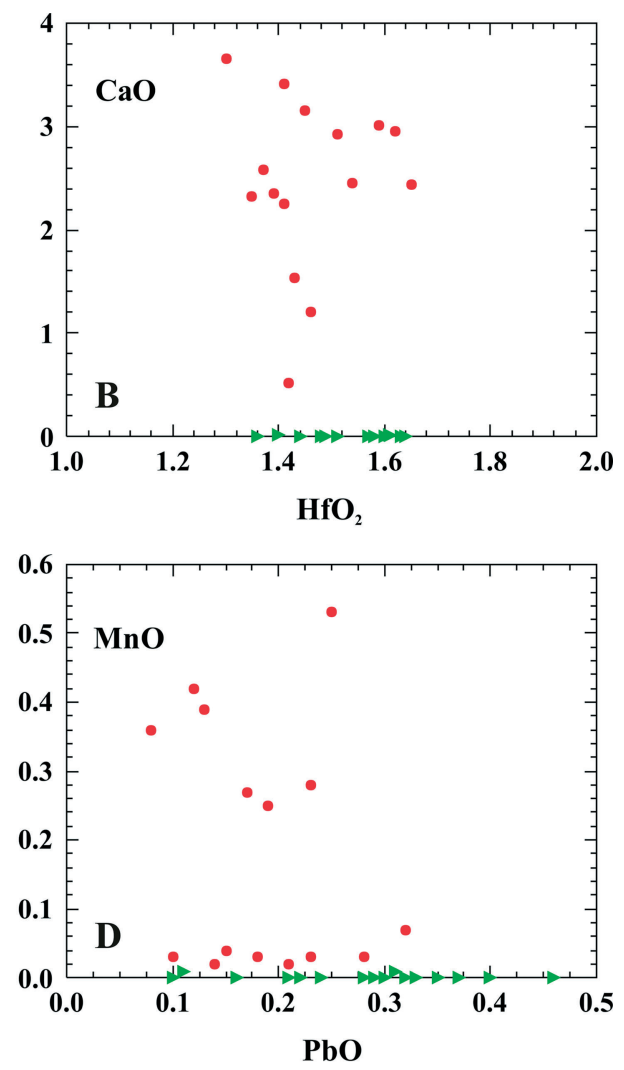

Fig. 14. Electron microprobe data on a zircon grain from the Susivaara sample A865 (for location of data points, see Fig. 5). 
vely (Fig. 14C). No systematic differences as a function of alteration have been observed in the concentrations of $\mathrm{HfO}_{2}\left(1.30-1.65\right.$ wt. \%), $\mathrm{TiO}_{2}(0.03-$ 0.09 wt. \%), and $\mathrm{PbO}\left(0.08-0.46\right.$ wt.\%). The $\mathrm{UO}_{2}$ content $(0.00-0.35 \mathrm{wt} . \%)$ is often lower in altered domains but in some spots it is higher than in fresh domains.

SEM-EDS analysis was used to identify the mineralogy of the distinct, bright specks, from a few to $20 \mathrm{~mm}$ in size, which commonly occur within altered domains (Figs. 4, 5, 7, 9, 11, 12). This revealed mainly $\mathrm{Th}$ and $\mathrm{Si}(+\mathrm{U}, \mathrm{Zr}, \mathrm{Ca})$ indicating that these mineral grains are composed of thorite $\left(\mathrm{ThSiO}_{4}\right)$. A few of the high-BSE intensity crystals turned out to be lead selenide (clausthalite). Baddeleyite was also found (Fig. 9A). A detailed study on the numerous silicate inclusions in zircon grains has not yet been carried out, but the currently available data show the presence of Fe-rich chlorite and sphene.

In summary, based on our limited electron microprobe data, the degree of alteration in zircons seems to correlate positively with measured $\mathrm{CaO}$ and inferred $\mathrm{H}_{2} \mathrm{O}$ contents and less systematically with concentrations of $\mathrm{FeO}$ and $\mathrm{MnO}$. In additi- on, altered zircon domains often contain tiny thorite grains.

\subsection{Sm-Nd results}

\subsubsection{Intrusions from Central Lapland}

The three samples from the Haaskalehto intrusion that were used for our Sm-Nd study are relatively well-preserved gabbros. Some of the Sm-Nd isotopic analyses of Table 4 showing relatively large errors were made in the 1980s using an old technique and a non-commercial mass spectrometer (Huhma, 1986). Ten analyses including two whole rocks (duplicated analyses on both) and clinopyroxene, amphibole and plagioclase separates define an isochron which gives an age of $2119 \pm 40 \mathrm{Ma}$ with an initial $\varepsilon_{\mathrm{Nd}}$ value of +0.8 . The relatively large MSWD value of 2.5 suggests some scatter in excess of analytical error. If the two plagioclase analyses are excluded, MSWD decreases to 0.9 and the age from the slope, as defined largely by the pyroxene analyses of sample 19-HSP-78, is $2187 \pm 44 \mathrm{Ma}$ $\left(\varepsilon_{\mathrm{Nd}}=+0.7\right.$, Fig. 15). This age is more consistent with the U-Pb zircon age of $2220 \pm 11 \mathrm{Ma}$ (Tyrväinen, 1983).

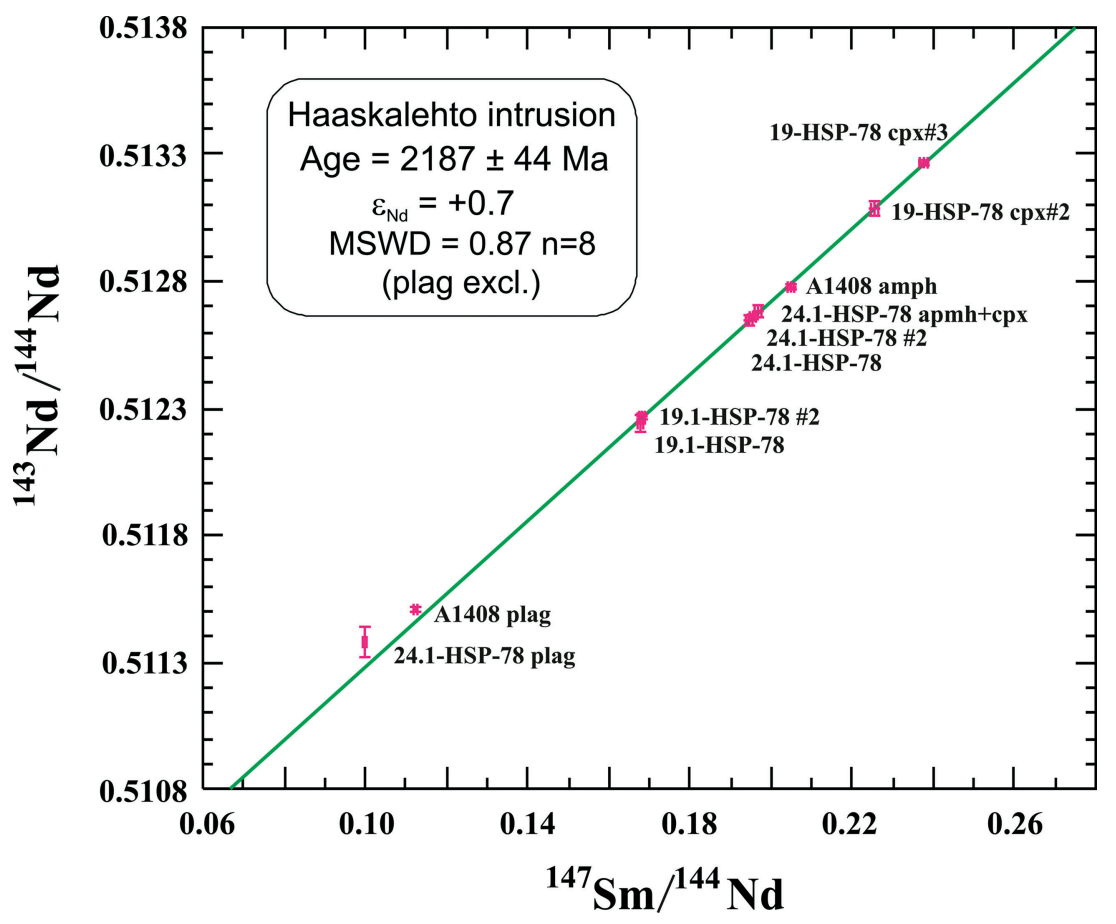

Fig. 15. Sm-Nd isotope data for whole-rock samples (gabbro) and mineral separates from the Haaskalehto intrusion. Analytical results on plagioclase are excluded in regression. 
Table 4. Sm-Nd isotopic data on $2.22 \mathrm{Ga}$ mafic intrusions $\left(\varepsilon_{\mathrm{Nd}}\right.$ calculated at $\left.2200 \mathrm{Ma}\right)$.

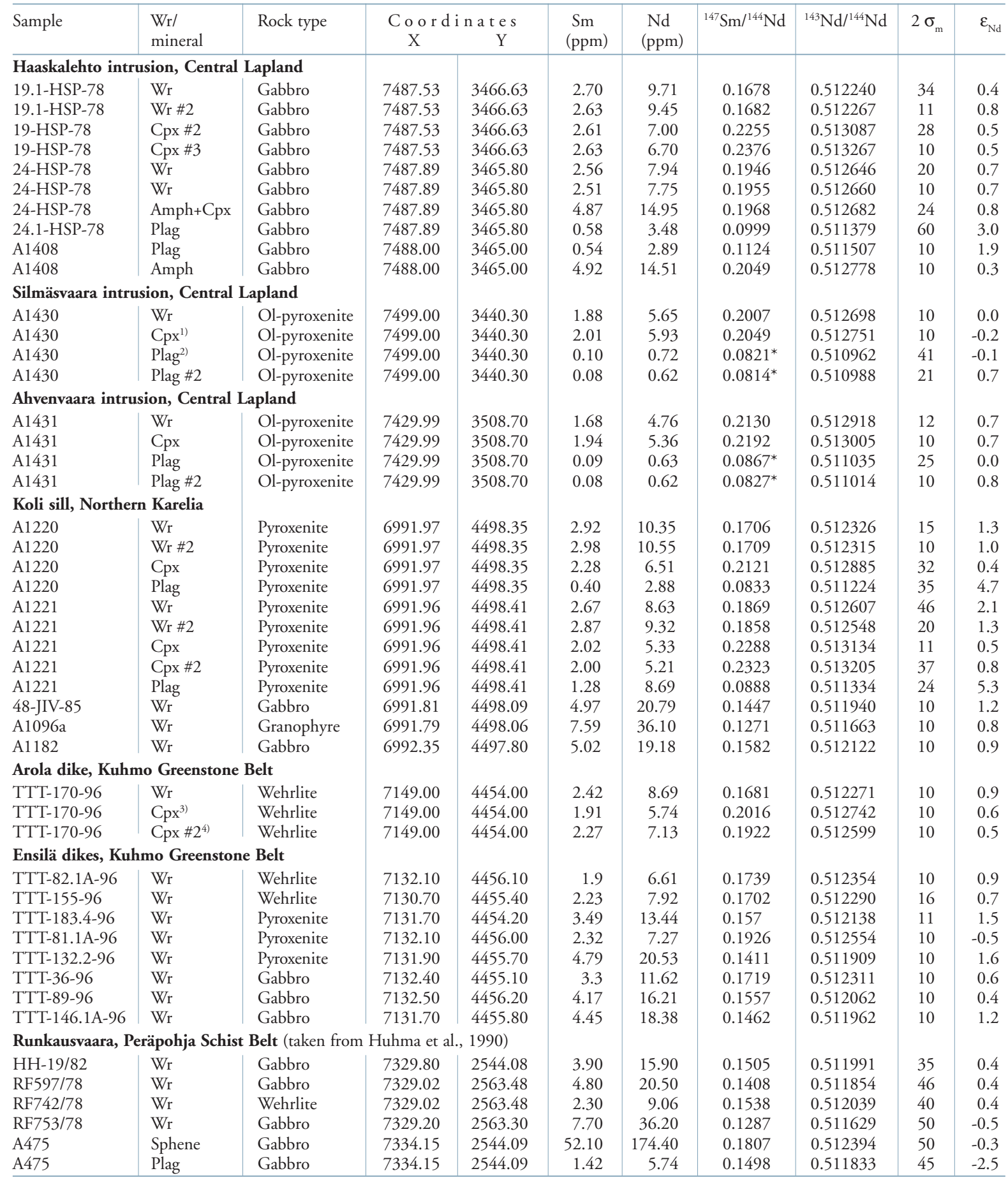

Appreviations: $\mathrm{Wr}=$ whole rock, $\mathrm{Plag}=$ plagioclase, $\mathrm{Cpx}=$ clinopyroxene, $\mathrm{Amph}=$ amphibole, $\mathrm{Ol}=$ olivine.

Notes on mineral separates: 1) density 3.3-3.4;2) density 3.63-2.76;3) magnetic fraction, density 3.25-3.33; 4) not handpicked, abrasion 30 min. The name of the rock type corresponds to the original, unmetamorphosed rock.

Error in ${ }^{147} \mathrm{Sm} /{ }^{144} \mathrm{Nd}$ is $0.4 \%$, except when marked by ${ }^{*}$, it is $2 \%$.

${ }^{143} \mathrm{Nd} /{ }^{144} \mathrm{Nd}$ radio is normalized to ${ }^{146} \mathrm{Nd} /{ }^{144} \mathrm{Nd}=0.7219$, error is 2 standard error of the mean in the last sign. digits.

Error in $\mathrm{e}_{\mathrm{Nd}}$ is normally \pm 0.4 units.

Coordinates are presented in the Finnish kkj-coordinate system. 
Microscopic observations reveal that plagioclase is turbid and yellowish and contains impurities, which were not possible to be removed by handpicking. It is conceivable that metamorphic effects, which are common in Lapland, have also slightly influenced the $\mathrm{Sm}-\mathrm{Nd}$ system in plagioclase in spite of the relatively well-preserved nature of the rocks at the sampling site.

Mineral separation using well-preserved olivine pyroxenites from the Silmäsvaara and Ahvenvaara intrusions yielded clean fractions of pyroxene and plagioclase. Due to low concentrations of Sm and $\mathrm{Nd}$, the analytical error for plagioclase is relatively large (Table 4). Four analyses from the Silmäsvaara sample A1430 give an age of $2185 \pm 35 \mathrm{Ma}\left(\varepsilon_{\mathrm{Nd}}=\right.$ 0.0, MSWD = 1.07), and the result from Ahvenvaara (A1431) is $2231 \pm 27 \mathrm{Ma}\left(\varepsilon_{\mathrm{Nd}}=+0.8\right.$, MSWD $=1.5$, Fig. 16). These ages are largely based on plagioclase, since the $\mathrm{Sm} / \mathrm{Nd}$ ratio in the whole-rock samples is close to that of pyroxene. Compared to Haaskalehto discussed above, plagioclase in the Silmäsvaara and Ahvenvaara samples is very clear and has likely remained closed since its magmatic crystallization. The age of c. $2.2 \mathrm{Ga}$ is compatible with the U-Pb zircon age of $2222 \pm 6 \mathrm{Ma}$ from the Har- junoja gabbro (Räsänen \& Huhma, 2001), which according to the aeromagnetic map, probably belonged originally to the same intrusive body as the Ahvenvaara intrusion.

\subsubsection{Dikes in the Kuhmo Greenstone Belt}

A few Sm-Nd analyses have been made on mafic dikes occurring in the Ensilä and Arola areas in the Archean Kuhmo Greenstone Belt. The samples are classified into three groups, wehrlites, metapyroxenites and metagabbros, which all contain largely a metamorphic mineral paragenesis. The results of the $\mathrm{Sm}-\mathrm{Nd}$ isotope analyses are shown in Table 4. The data are scattered probably due to metamorphic effects (Fig. 17). An attempt was made to use pyroxene to date a wehrlitic sample (TTT-170-96) from Arola, but no pure fraction was possible to obtain due to alteration of pyroxene. Nevertheless, two analyses were made on altered pyroxene concentrates, which together with whole-rock data yield a date of $2120 \pm 86 \mathrm{Ma}$. The average $\varepsilon_{\mathrm{Nd}}(2220 \mathrm{Ma})$ value of +0.7 for the whole data set is similar to that of other GWA intrusions (see below).

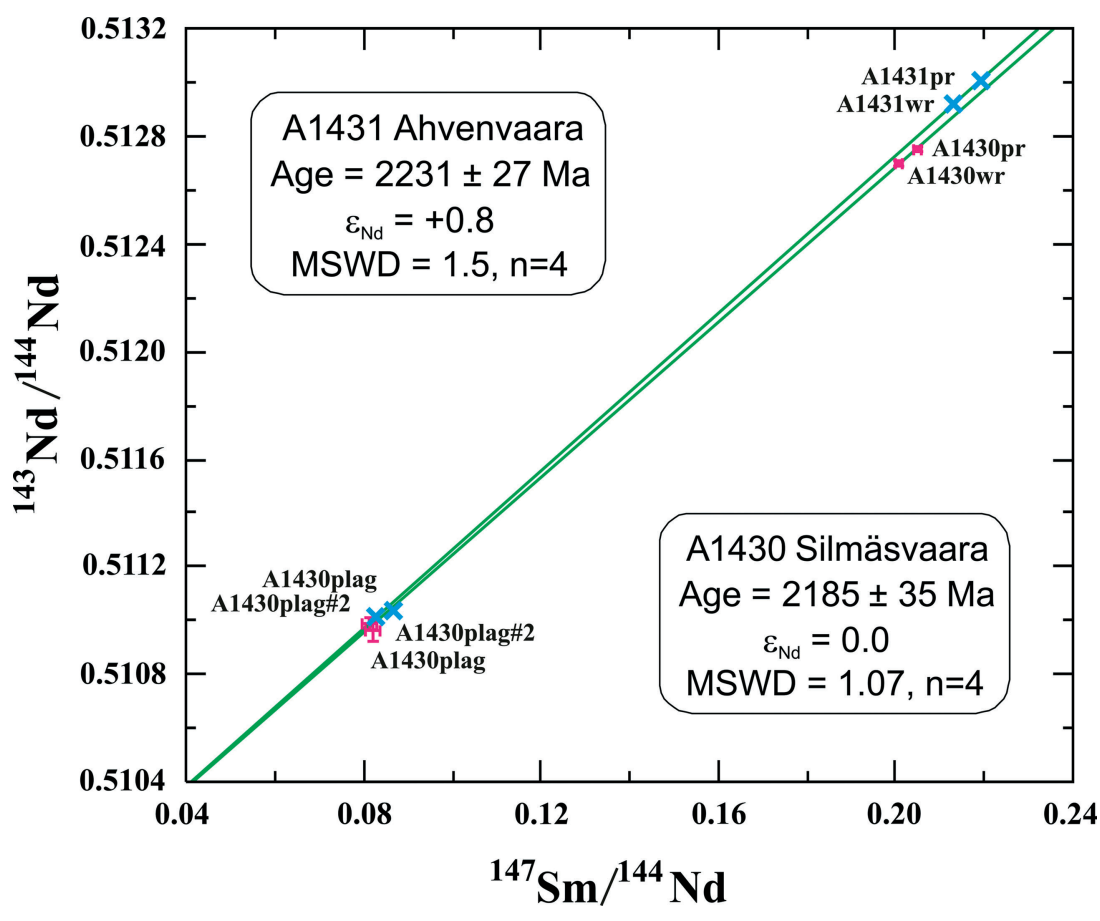

Fig. 16. Sm-Nd isotope data for the Silmäsvaara (A1430) and Ahvenvaara (A1431) intrusions. Analytical results on olivine pyroxenites and clinopyroxene and plagioclase separates are regressed separately for both intrusions. 


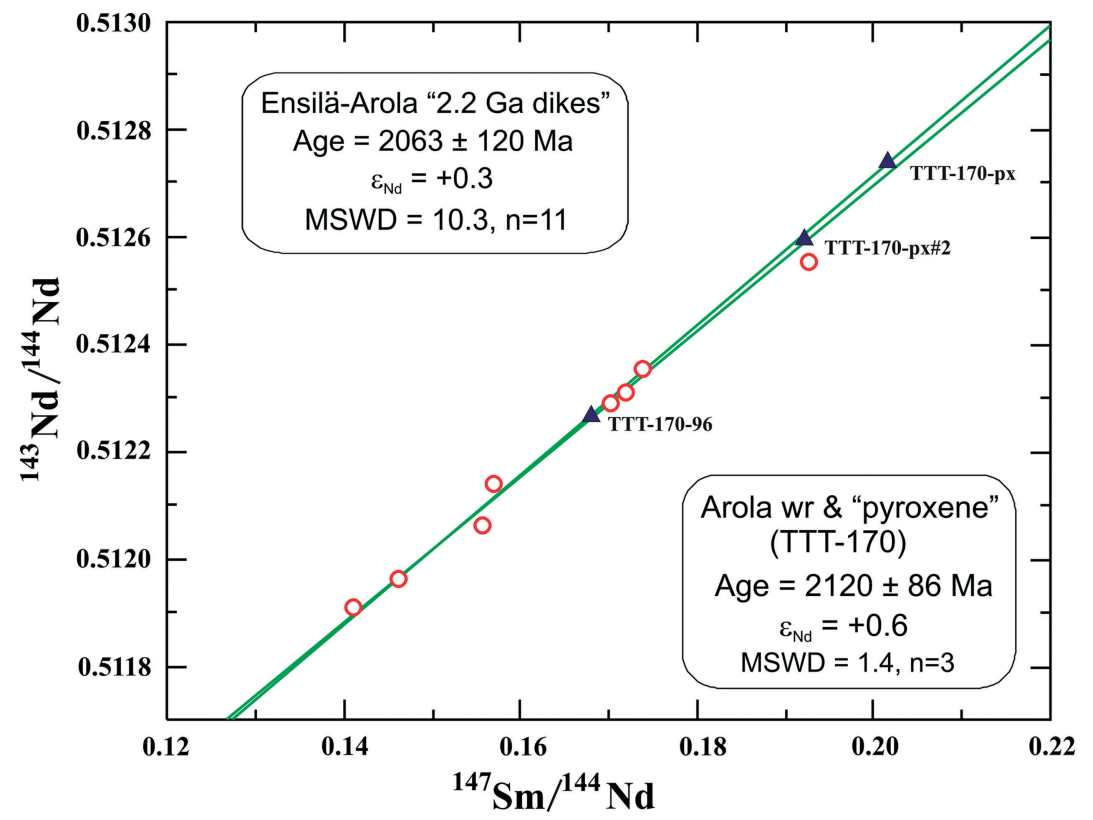

Fig. 17. Sm-Nd isotope data for Paleoproterozoic mafic intrusive rocks from the Archean Kuhmo Greenstone Belt. Samples include two impure clinopyroxene separates and one wehrlitic whole-rock sample from Arola and ultramafic and mafic whole-rock samples from Ensilä. Two regression lines are shown, one based on the Arola analyses and the other on the whole data set.

\subsubsection{Koli sill (A1220, A1221)}

The Sm-Nd studies from the 300-m-thick Koli layered sill were focused on the Kaunisniemi section described previously by Vuollo (1988). The mineral separation from two pyroxenites yielded fairly clean fractions of clinopyroxene, but albitic plagioclase is generally turbid and yellowish. The Sm-Nd data are shown in Table 4, which also includes some older analyses having relatively large errors. The twelve analyses available on five whole rocks and mineral fractions do not define an isochron, as shown by the calculated MSWD value of 30 . If the two analyses on albitic plagioclase are excluded, an age of $2201 \pm 58 \mathrm{Ma}$ and an initial $\varepsilon_{\mathrm{Nd}}$ value of + 1.0 can be calculated (Fig. 18). The slightly elevated MSWD value of 3.5 may be due to underestimation of the analytical error (in old analyses), inclusion of samples from several locations in the same regression or most likely due to opening of the Sm$\mathrm{Nd}$ system during metamorphism. The metamorphic effects on albitic plagioclase are obvious, as can be seen from the $\mathrm{Sm}-\mathrm{Nd}$ age estimate of c. 1.9 Ga calculated for the whole rock-plagioclase pairs of samples A1220 and A1221 (Fig. 18).

The analysis on granophyre provides an initial $\varepsilon_{\mathrm{Nd}}(2220 \mathrm{Ma})$ value of +0.8 which is similar to the cumulates of the main series. This suggests that the granophyre in the upper part of the sill was formed from evolved magma without any significant contamination from the adjacent Archean country rocks.

\section{Discussion and conclusions}

\subsection{Timing of the GWA magmatism}

The previous age determinations using the conventional U-Pb method have yielded dates from 1.97 to $2.22 \mathrm{Ga}$ for intrusions that have petrological characteristics and field relationships typical of the gabbro-wehrlite association. This age range probably reflects the degree of preservation of the dated zircons and the GWA magmatism was not such a longlived event (Hanski et al., 2001), but rather could have been even shorter in duration than the resolution of our dating methods. To reach this conclusion has required a large number of conventional zircon analyses, supplemented by occasional baddeleyites, from various parts of eastern and northern Finland. The new data acquired by the SIMS techni- 


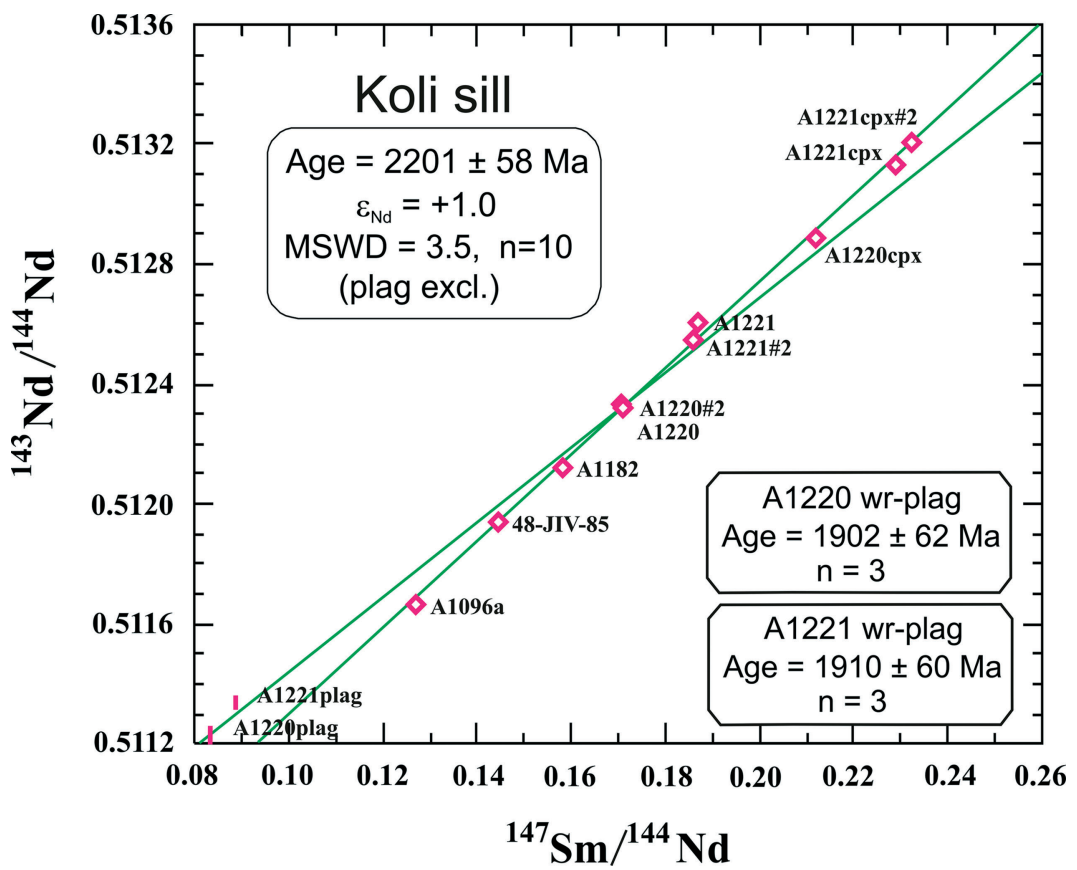

Fig. 18. Sm-Nd isotope data for samples $\mathrm{A} 1220$ and $\mathrm{A} 1221$ from the Koli sill. A linear regression of analyses on whole-rock samples and clinopyroxene separates gives an isochron age of $2201 \pm 58 \mathrm{Ma}$, while plagioclase-whole rock pairs yield ages of c. 1900 Ma. que, which is able to circumvent problems caused by zircon alteration, has confirmed this view. These results together with BSE images and electron microprobe analyses provide us now with a clearer understanding of the large variation of the U-Pb isotopic disturbance in zircon shown by the previous ID-TIMS studies. The most well-preserved zircon grains are found in the GWA intrusions in Central Lapland, and hence the zircon U-Pb ages obtained for the Haaskalehto and Harjunoja intrusions, $2220 \pm 11 \mathrm{Ma}$ and $2222 \pm 6 \mathrm{Ma}$ (Tyrväinen, 1983; Räsänen \& Huhma, 2001), can be taken as the most representative determinations of the timing of the GWA magmatism in Finland.

\subsection{Alteration of zircon by fluids}

Several mechanisms have been proposed to control the degree of $\mathrm{U}-\mathrm{Pb}$ isotopic discordance in zircon (see the review by Gebauer and Grünenfelder, 1979). One of these is low-temperature hydrothermal alteration of radiation-damaged zircon as originally put forward by Krogh and Davis (1975). Several lines of evidence make the alteration model most appealing in our case, including the high contents of non-formula components $\left(\mathrm{CaO}, \mathrm{Al}_{2} \mathrm{O}_{3}\right.$,
$\mathrm{FeO}, \mathrm{MnO}, \mathrm{H}_{2} \mathrm{O}$ ) in zircon grains, their inferred hydrous nature, the general lack of magmatic zoning features (cf. Connelly, 2001), and other physical indications of strong alteration. In Fig. 19, our ion microprobe analyses have been divided into two groups on the basis of the BSE intensity of the analyzed spots. There is an obvious relationship between the average atomic number of zircon (degree of hydration and related alteration) and the degree of discordance as the domains displaying low BSE intensities have experienced the largest amount of $\mathrm{Pb}$ loss. Note that a high BSE intensity (light shade) does not necessarily indicate a non-altered nature of zircon. Recrystallized parts of the grains may be even more light-colored than pristine zones. This is well exemplified by Figs. 7F and 12E.

Geisler and Schleicher (2000) have studied the relationship between the chemical composition of some non-metamorphosed zircons and their age discordance. They observed a negative correlation between the $\mathrm{CaO}$ content and apparent U-Thtotal $\mathrm{Pb}$ ages with the highest, concordant ages obtained for those zircon domains that had $\mathrm{CaO}$ contents of less than $0.2 \mathrm{wt} . \%$. In contrast, the dates yielded by the domains having elevated $\mathrm{CaO}$ concentrations were drastically lower than the accepted 


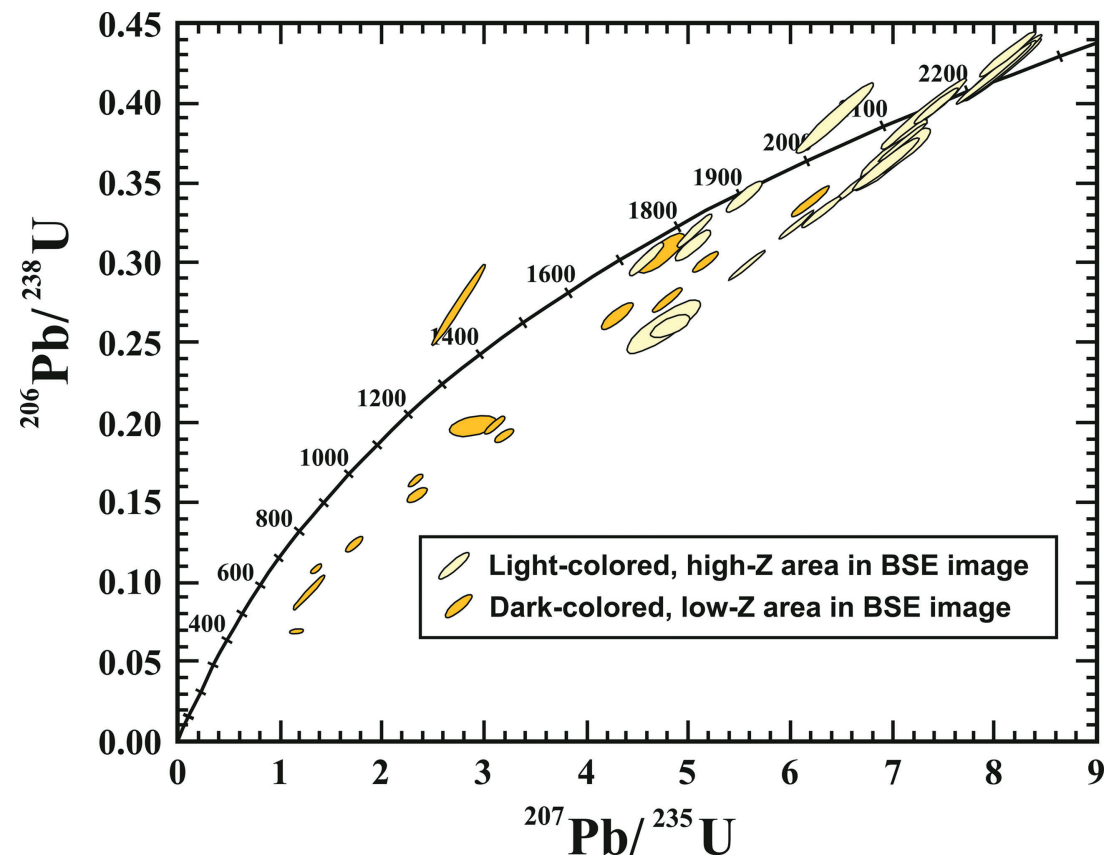

Fig. 19. Concordia plot of the SIMS U-Pb data divided into two groups on the basis of the BSE intensity of the analyzed spot. magmatic ages. Geisler and Schleicher (2000) attributed the high contents of $\mathrm{Ca}$ and other non-formula elements to ion exchange processes during fluid-induced alteration processes that led to removal of $\mathrm{Zr}, \mathrm{Si}$ and $\mathrm{Pb}$ from the zircon lattice. Later Geisler et al. (2001, 2002, 2003) demonstrated experimentally that in a large temperature range between $175^{\circ} \mathrm{C}$ and $650^{\circ} \mathrm{C}$, hydrothermal alteration of partially metamict zircon through an interaction of $\mathrm{CaCl}_{2}$-bearing solutions can generate zircon domains with elevated $\mathrm{CaO}$ contents up to c. 2 wt.\%. In these experiments, addition of $\mathrm{CaO}$ was accompanied by extensive hydration of the mineral with resultant $\mathrm{H}_{2} \mathrm{O}$ contents reaching 8 wt.\%. Both experimental data and natural examples show that, due to the incompatibility of $\mathrm{Pb}^{2+}$ in the newly grown zircon phase, radiogenic $\mathrm{Pb}$ is easily rejected from the mineral grains during hydrothermal annealing of partially metamict zones, resulting in a strong disturbance of the $\mathrm{U}-\mathrm{Pb}$ isotope system (Pidgeon et al., 1966; 1973; Krogh and Davis, 1975; Geisler et al., 2003a,b).

Based on our electron microprobe and SIMS data from a single zircon grain from the Susivaara sill, the same kind of relationship between the $\mathrm{CaO}$ content and discordance, as observed by Geisler and
Schleicher (2000), may be a general feature of altered zircon grains in the GWA intrusions. We suggest that the high $\mathrm{CaO}$ contents and high degree of discordance are related to hydrothermal alteration of the zircon grains by $\mathrm{CaCl}_{2}$-bearing fluids and the low analytical totals of the electron microprobe analyses reflect high $\mathrm{H}_{2} \mathrm{O}$ contents of the altered zircon domains.

Apart from the loss of radiogenic $\mathrm{Pb}$ and gain of $\mathrm{Ca}$, hydrothermal alteration of metamict zircon can result in significant changes in concentrations of other minor or trace elements such as $\mathrm{Sr}, \mathrm{Ba}, \mathrm{Al}$, Fe, Mn, Y, REE, and most importantly from the point of view of geochronology, $U$ and Th (Krogh and Davis, 1975; Geisler et al., 2003b). The behavior of REE and Y during the alteration processes may be complicated. For example, Geisler et al. (2003b) observed a positive correlation between the $\mathrm{CaO}$ and $\mathrm{Y}_{2} \mathrm{O}_{3}$ contents in natural altered zircon grains from an Egyptian granite, whereas in our study, the altered domains show an $\mathrm{Y}_{2} \mathrm{O}_{3}$ content of about a half of that observed in the least altered domains (Fig. 14C). Evidently, a more comprehensive electron microprobe and LA-ICP-MS study of zircon in the GWA rocks is needed.

High $U$ and Th contents induce high a-decay 
doses and high degrees of metamictization and, consequently, the altered zircon grains may exhibit a positive correlation between the $\mathrm{U}$ and Th contents and the degree of $\mathrm{U}-\mathrm{Pb}$ isotopic discordance. Figure 20 shows $U$ concentrations as a function of ${ }^{207} \mathrm{~Pb} /$ ${ }^{235} \mathrm{U}$ as determined by SIMS. Using the ${ }^{207} \mathrm{~Pb} /{ }^{235} \mathrm{U}$ ratio as an index of discordance, it can be seen that there is no clear correlation between $U$ content and degree of discordance. The same applies to the Th content and $\mathrm{Th} / \mathrm{U}$ which varies widely from lower than 1.0 to higher than 10 . Identification of independent Th-rich mineral grains (thorite) in the lowintensity BSE zircon domains is noteworthy in this context as their presence indicates secondary mobility of Th and within the spot of the beam, they may strongly affect the $\mathrm{Th} / \mathrm{U}$ ratio and Th concentration in the analytical results. The lack of correlation between the $U$ content and ${ }^{207} \mathrm{~Pb} /{ }^{235} \mathrm{U}$ in severely altered zircons was regarded by Cherniak and Watson (2000) as an indication of non-diffusional processes, such as recrystallization and various fluidassisted processes that could significantly alter the $\mathrm{Pb}$ isotope ratios and $\mathrm{U}$ content in zircon. It is noteworthy that even though major loss of radiogenic $\mathrm{Pb}$ from zircons and consequent decrease in ${ }^{207} \mathrm{~Pb} /$ ${ }^{235} \mathrm{U}$ and ${ }^{206} \mathrm{~Pb} /{ }^{238} \mathrm{U}$ seem to have taken place in our case, hydrothermal alteration may also have lead to gain or loss of $U$ and/or Th.

As plagioclase in the GWA intrusions is almost ubiquitously albitized, the source of $\mathrm{CaO}$ of the altered zircons could be envisaged to be the anorthite component of plagioclase liberated during albitization. This is consistent with the most well-preserved and concordant zircon being found in the sample from the Haaskalehto intrusion, one of the GWA intrusions that have a relatively well-preserved primary magmatic mineralogy including labradoritic plagioclase. The $\mathrm{CaO}$ released during albitization of plagioclase could also have participated in the change of ilmenomagnetite to sphene, a phenomenon that is often observed in the GWA sills. Alteration of ilmenomagnetite was accompanied by liberation of Fe, which may have been partly consumed in the formation of secondary biotite, but could also have been incorporated into other secondary minerals including altered domains of zircon grains.

The more or less one-stage process described above may seem reasonable, but it is not easily fit with the observed U-Pb isotopic systematics of zircon and sphene. Taken together, the ID-TIMS and SIMS analyses suggest a multi-episodic history for the rocks. First, concordant ID-TIMS sphene ages

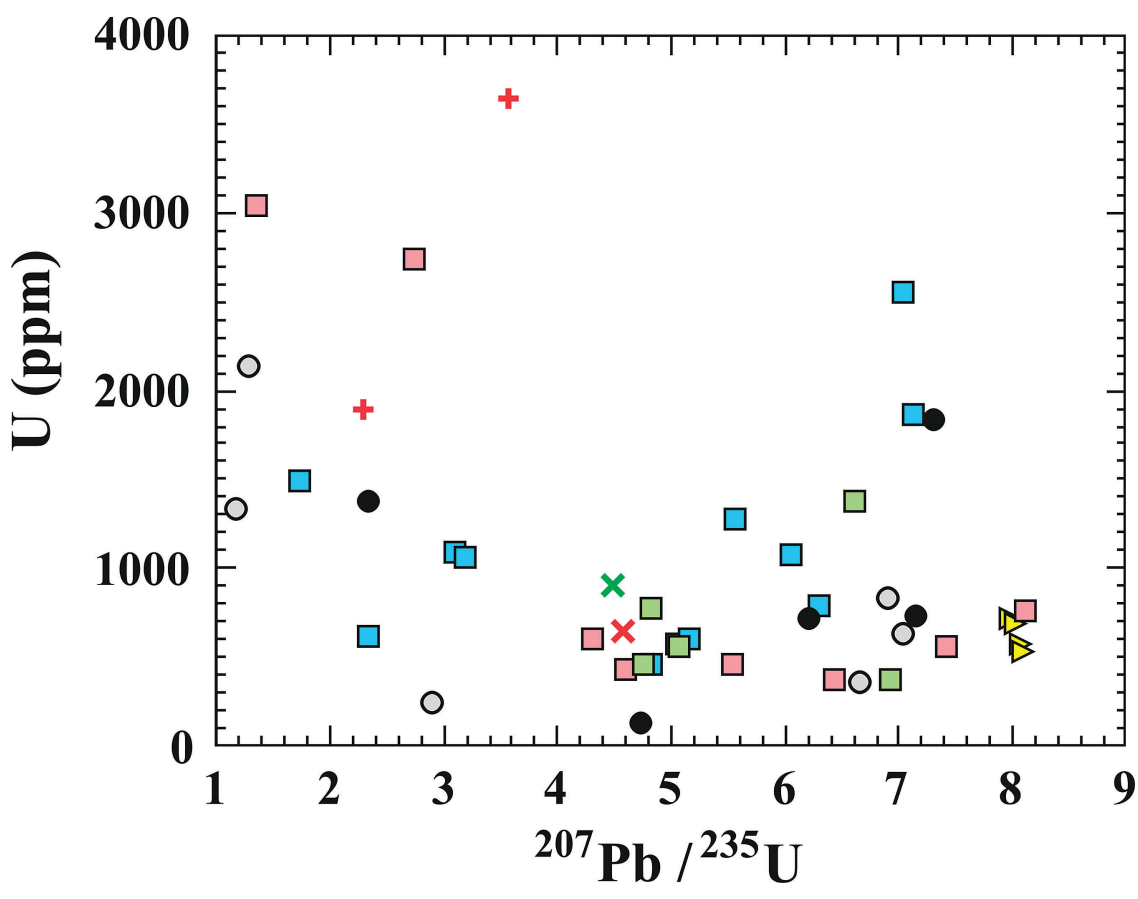

Fig. 20. Uranium - ${ }^{207} \mathrm{~Pb} /{ }^{235} \mathrm{U}$ relations for zircon grains as determined by ion microprobe. ${ }^{207} \mathrm{~Pb} /{ }^{235} \mathrm{U}$ is used as an index of discordance. 
of c. 2.2 Ga indicate that some alteration phenomena were very early and took place perhaps already in the late-magmatic stage, though we must add that in the GWA intrusions sphene is not exclusively concordant at $2.2 \mathrm{Ga}$ but partially suffered from later isotopic disturbances together with zircon (see figure 3 in Hanski et al. 2001). Second, some SIMS ages on zircon are nearly concordant at $1.8-1.9 \mathrm{Ga}$ suggesting a resetting event related to the Svecofennian orogeny, which is also supported by our $\mathrm{Nd}$ isotopic data on plagioclase from Koli and some other zircon studies from northern Finland (Mertanen et al., 1989). Third, some zircon grains give very discordant SIMS results indicative of a very recent $\mathrm{Pb}$ loss as these minerals contain sufficient uranium to have developed higher ${ }^{206} \mathrm{~Pb} /{ }^{238} \mathrm{U}$ and ${ }^{207} \mathrm{~Pb} /{ }^{235} \mathrm{U}$ ratios over time had the major $\mathrm{Pb}$ loss occurred much earlier.

\subsection{Alteration event}

Given a multi-episodic history of zircon in the GWA intrusions, the large scatter in the lower intercept ages of the discordias obtained in this and previous studies becomes understandable. These ages range from c. 150 to $960 \mathrm{Ma}$ and appear to be geologically meaningless, which is in accord with the view that the lower intercept ages in general have no geological significance (e.g. Mezger and Krogstad, 1997). However, lower intercept ages obtained from spot analysis of zircon that contain a high concentration of non-formula components (e.g. $\mathrm{CaO}$ ) can be sufficiently discordant (close to the lower intercept with concordia) to precisely date a leaching event and relate it to the regional thermotectonic history (Geisler et al., 2001). For example, Geisler et al. (2003b) obtained $100 \%$ discordant SHRIMP ages of c. $20 \mathrm{Ma}$ for hydrothermally altered, Ca-bearing zircons from a c. 620 Ma Egyptian granite mentioned above. They were able to link this alteration event and similar fission track ages of apatite to the mafic magmatism associated with the main rifting phase of the Red Sea. The most discordant analytical points of our SIMS study are not sufficiently close to the concordia to yield a precise time for the suggested relatively recent $\mathrm{Pb}$ loss event, but nevertheless indicate a Paleozoic age of some hundred million years.

It is worth noting that even though the variation in discordance shown by our SIMS data is large, it is not larger than that displayed by the previously published zircon and baddeleyite ID-TIMS analyses from the GWA intrusions (Hanski et al., 2001, figure 2). Figure 21 exhibits all available SIMS and ID-TIMS data on a concordia diagram. The whole data set can be accounted for by various combinations of ancient and relatively recent $\mathrm{Pb}$ loss. The huge variation in the ${ }^{207} \mathrm{~Pb} /{ }^{235} \mathrm{U}$ and ${ }^{206} \mathrm{~Pb} /{ }^{238} \mathrm{U}$ ratios generates a long array that converges towards a lower intercept age of c. 300 Ma. Some ID-TIMS data points in this array even show a higher degree of discordance than the most discordant in situ SIMS analyses. With the help of the ion microprobe, zircon grains from these most discordant bulk samples could potentially be used to get more precise age constraints on the relatively recent $\mathrm{Pb}$ loss deduced from the present zircon data.

Larson and Tullborg (1998) drew attention to the fact that conventional U-Pb zircon data from the Svecofennian domain in Sweden often record late Paleozoic lower intercept ages, which fit with the timing of intense regional $\mathrm{Pb}$ mobilization. Similar lower-intercept ages are also common for Paleoproterozoic rocks further east in Finland as exemplified by ID-TIMS measurements from northern Finland in Fig. 22. Larson and Tullborg (1998) attributed the relatively recent lower-intercept ages in the crystalline basement of the shield to leaching of zircons by low-temperature hydrothermal fluids, whose generation was related to burial of the shield beneath upper Paleozoic sedimentary rocks produced by rapid erosion of the Caledonides. Högdahl et al. (2001) observed an almost complete resetting at c. $380 \mathrm{Ma}$ of Paleoproterozoic zircon in a highgrade deformation zone in central Sweden. They suggested that this annealing event affected mostly metamict zircon and was influenced by low-T saline fluids circulating in the basement due to the Caledonian orogeny. Still one reason for heating of the lithosphere of the Fennoscandian Shield in the Paleozoic time may have been the (mantle plumerelated?) magmatic event that generated Devonian 

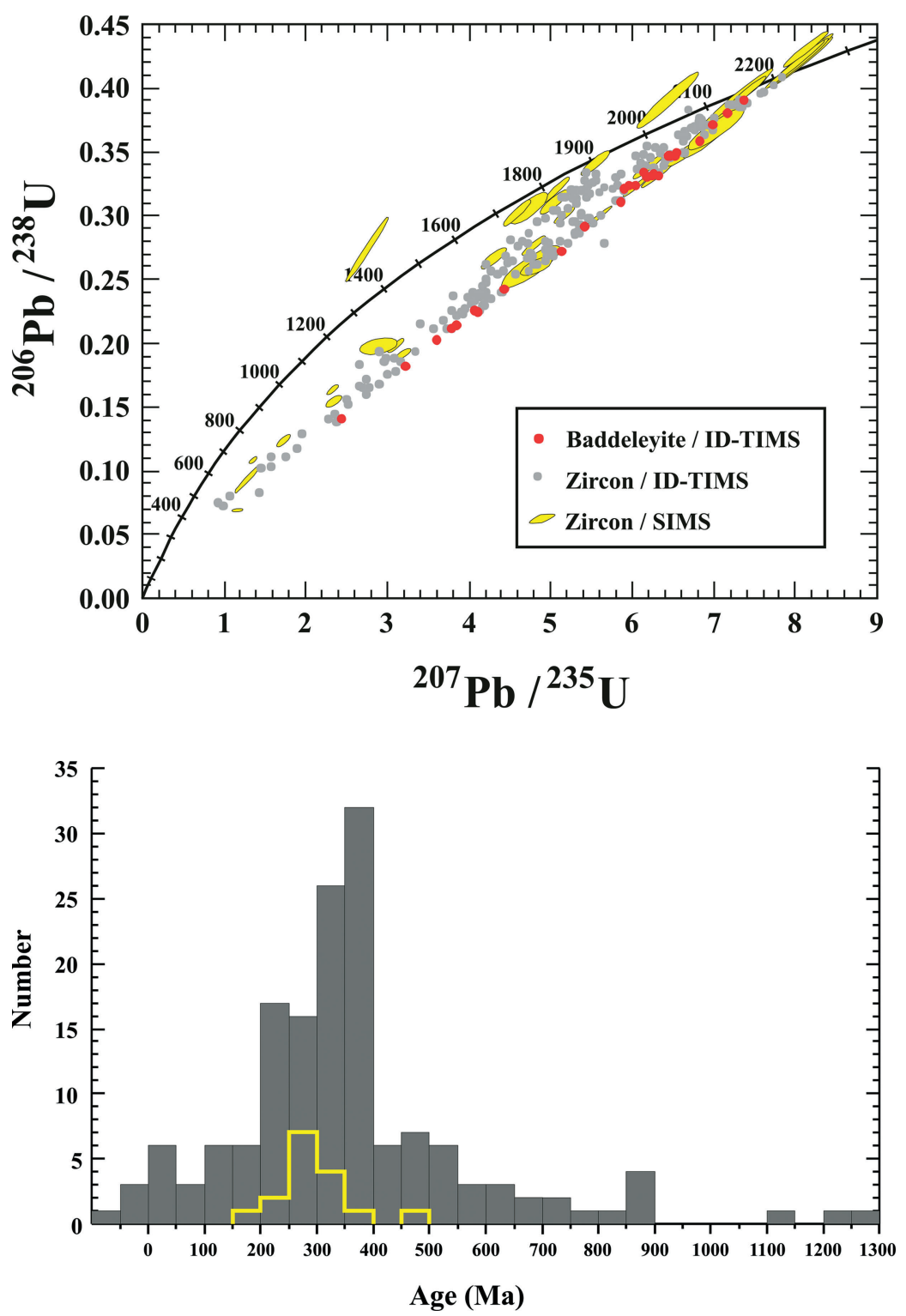

Fig. 21. Concordia plot of the U-Pb SIMS and ID-TIMS data on zircon and baddeleyite of this study and previous studies (after Hanski et al., 2001, figure 2).

Fig. 22. Concordia-based lower-intercept ages for ID-TIMS U-Pb zircon dates of Paleoproterozoic rocks from northern Finland. Also shown as a yellow histogram is the distribution of lower-intercept ages of U-Pb zircon data from the Svecofennian domain in Sweden as published by Larson and Tullborg (1998).

alkaline rocks in the eastern part of the shield (e.g. Downes et al., 2005). It is unclear whether any of the above-mentioned geological processes were responsible for the wide-spread resetting of the U$\mathrm{Pb}$ system in the zircons of the $2.2 \mathrm{Ga}$ GWA intrusions. Anyway, alteration and recrystallization of partially metamict zircon by hydrothermal solutions can take place at fluid temperatures lower than 200 ${ }^{\circ} \mathrm{C}$ and the former existence and timing of such a thermal event may not be easily verified with other isotopic methods than the $\mathrm{U}-\mathrm{Pb}$ zircon dating.

\subsection{Nd isotopic characteristics of the GWA magmatism}

Earlier Sm-Nd isotope data on the Runkausvaara sill published by Huhma et al. (1990) yielded an imprecise isochron with an age of $2205 \pm 220 \mathrm{Ma}$ and initial $\varepsilon_{\mathrm{Nd}}$ of $0.1 \pm 1.4$. Our new, somewhat more precise isochron data give similar results with the initial $\varepsilon_{\mathrm{Nd}}$ values being consistently between 0.0 and +1.0 . Combining all relevant data of a total of 30 analyses gives an age of $2223 \pm 28 \mathrm{Ma}$ and an 
initial isotopic composition corresponding to $\varepsilon_{\mathrm{Nd}}$ of +0.6 . This age is in good agreement with the zircon data and the initial $\varepsilon_{\mathrm{Nd}}$ value is thought to be close to that of the parental magma. Figure 23 shows a diagram of calculated initial $\varepsilon_{\mathrm{Nd}}(2220 \mathrm{Ma})$ values vs. $\mathrm{Sm} / \mathrm{Nd}$ ratios for mineral and whole-rock samples, constructed using data from the present study and Huhma et al. (1990). It is evident that plagioclase data are very scattered, while pyroxenes and most whole-rock samples give initial $\varepsilon_{\mathrm{Nd}}$ values close to the above mentioned isochron-based, preferred value of +0.6 .

The initial $\mathrm{Nd}$ isotopic composition of the GWA magma with a slightly positive initial $\varepsilon_{\mathrm{Nd}}(2220 \mathrm{Ma})$ differs from that of the contemporaneous depleted mantle reservoir $\left(\varepsilon_{\mathrm{Nd}}=-+3\right)$ as defined by the isotopic evolution of DePaolo (1981). This deviation may have resulted from interaction between the magma and crustal rocks. However, our $\mathrm{Nd}$ isotopic data as a whole indicate that the magma that produced the GWA intrusions in various parts of northern and eastern Finland was isotopically homogeneous and hence we conclude that it did not undergo significant upper crustal contamination upon emplacement and subsequent fractional crystallization.

\section{Acknowledgements}

The Nordic geological ion microprobe facility (NORDSIM) is operated and funded under an agreement between the respective research funding agencies of Denmark, Norway and Sweden, the Geological Survey of Finland, and the Swedish Museum of Natural History. Martin Whitehouse is thanked for assistance in the SIMS work and Bo Johanson and Zhenyu Luo for performing the electron microprobe analyses. We acknowledge the helpful reviews by Stefan Claesson, Kåre Kullerud and Arto Luttinen. This paper is NORDSIM contribution No. 257.

\section{References}

Cherniak, D.J. \& Watson, E.B. 2000. Pb diffusion in zircon. Chemical Geology 172, 5-24.

Connelly, J.N. 2001. Degree of preservation of igneous zonation in zircon as a signpost for concordance in $\mathrm{U} / \mathrm{Pb}$ geochronology. Chemical Geology 172, 25-39.

DePaolo, D.J. 1981. Neodymium isotopes in the Colorado

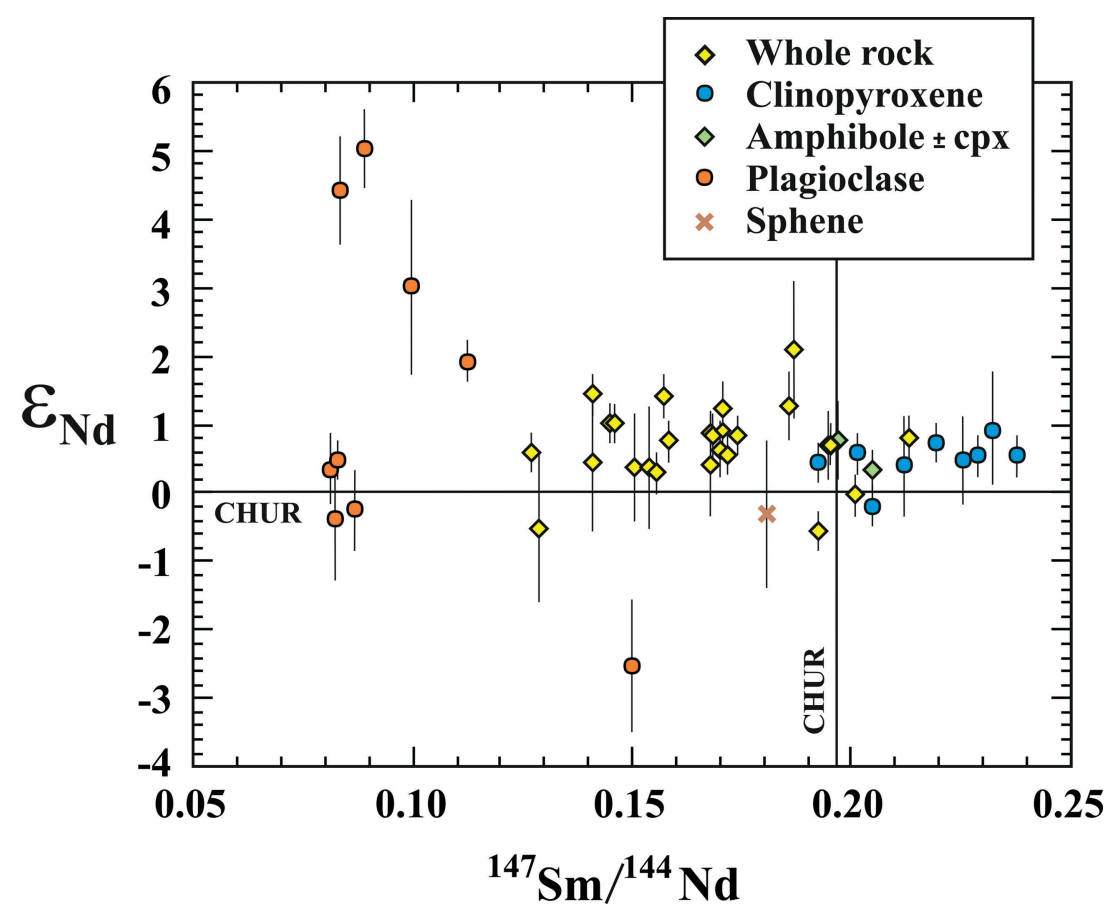

Fig. 23. $\varepsilon_{\text {Nd }}(2220 \mathrm{Ma})$ vs. ${ }^{147} \mathrm{Sm} /$ ${ }^{144} \mathrm{Nd}$ diagram for whole-rock and mineral analyses. Data from this study and Huhma et al. (1990). 
Front Range and crust-mantle evolution in the Proterozoic. Nature 291, 684-687.

Downes, H., Balaganskaya, E., Beard, A., Liferovich, R. \& Demaiffe, D. 2005. Petrogenetic processes in the ultramafic, alkaline and carbonatitic magmatism in the Kola Alkaline Province: a review. Lithos 85, 48-75.

Evins, P.M. \& Laajoki, K. 2001. Age of the Tokkalehto metagabbro and its significance to the lithostratigraphy of the early Proterozoic Kuusamo supracrustal belt, northern Finland. Bulletin of the Geological Society of Finland 73, 5-15.

Gebauer, D. \& Grünenfelder, M. 1979. U-Th-Pb dating of minerals. In: Jäger, E. \& Hunziker, J.C. (eds.) Lectures in Isotope Geology. Springer-Verlag, Berlin, pp. 105-131.

Geisler, T. \& Schleicher, H. 2000. Improved U-Th-total Pb dating of zircons by electron microprobe using a simple new background modeling procedure and $\mathrm{Ca}$ as a chemical criterion of fluid-induced U-Th-Pb discordance in zircon. Chemical Geology 163, 269-285.

Geisler, T., Ulonska, M., Schleicher, H., Pidgeon, R.T. \& van Bronswijk, W. 2001. Leaching and differential recrystallization of metamict zircon under experimental hydrothermal conditions. Contributions to Mineralogy and Petrology 141, 53-65.

Geisler, T., Pidgeon, R.T., van Bronswijk, W. \& Kurtz, R. 2002. Transport of uranium, thorium, and lead in metamict zircon under low-temperature, hydrothermal conditions. Chemical Geology 191, 141-154.

Geisler, T., Pidgeon, R.T., Kurtz, R., van Bronswijk, W. \& Schleicher, H. 2003a. Experimental hydrothermal alteration of partially metamict zircon. American Mineralogist 88, 1496-1513.

Geisler, T., Rashwan, A.A., Rahn, M.K.W., Poller, U., Zwingmann, H., Pidgeon, R.T., Schleicher, H. \& Tomaschek, F. 2003b. Low-temperature hydrothermal alteration of natural metamict zircons from the Eastern Desert, Egypt. Mineralogical Magazine 67, 485-508.

Hanski, E. 1982. Albiittidiabaasit ja niihin liittyvät ultramafiset kivet Kuhmon ja Kolin alueilla. Summary: A comparative study of albite diabases and related ultramafic rocks in the Kuhmo and Koli areas, eastern Finland. Arkeeisten alueiden malmiprojekti, Raportti No. 6. Oulun yliopisto, $75 \mathrm{p}$.

Hanski, E. 1984. Geology of the gabbro-wehrlite association in the eastern part of the Baltic Shield. Arkeeisten alueiden malmiprojekti, Raportti No. 20. Oulun yliopisto, 78 p.

Hanski, E. 1986a. The gabbro-wehrlite association in the eastern part of the Baltic Shield. In: Fiedrich, G.H., et al. (eds.) Geology and Metallogeny of Copper Deposits. Springer-Verlag, Berlin Heidelberg, pp. 151-170.

Hanski, E. 1986b. Intrusions of the gabbro-wehrlite association and their stratigraphic implications in Finland. In: Sokolov, V.A. \& Heiskanen, K.I. (eds.) Early Proterozoic of the Baltic Shield. Proceedings of the Finnish-Soviet
Symposium held in Petrozavodsk 19th-27th August, 1985. Karelskii Filial AN SSSR, Petrozavodsk, pp. 123136.

Hanski, E. 1987. Differentiated albite diabases gabbro-wehrlite other association. In: Aro, K. \& Laitakari, I. (eds.) Diabases and Report mafic mafic dyke rocks in Finland, Geological Survey of Finland, Report of Investigations 76, 35-44 (in Finnish, English abstract).

Hanski, E., Huhma, H. \& Vaasjoki, M. 2001. Geochronology of northern Finland: a summary and discussion. In: Vaasjoki, M. (ed.) Radiometric Age Determinations from Finnish Lapland and Their Bearing on the Timing of Precambrian Volcano-Sedimentary Sequences. Geological Survey of Finland, Special Paper 33, 255-279.

Högdahl, K., Gromet, L.P. \& Broman, C. 2001. Low P-T Caledonian resetting of U-rich Paleoproterozoic zircons, central Sweden. American Mineralogist 86, 543-546.

Huhma, H. 1986. Sm-Nd, U-Pb and Pb-Pb isotopic evidence for the origin of the early Proterozoic Svecokarelian crust in Finland. Geological Survey of Finland, Bulletin 337, $1-52$.

Huhma, H., Cliff, R.A., Perttunen, V. \& Sakko, M. 1990. Sm$\mathrm{Nd}$ and $\mathrm{Pb}$ isotopic study of mafic rocks associated with early Proterozoic continental rifting: the Peräpohja schist belt in northern Finland. Contributions to Mineralogy and Petrology 104, 367-379.

Huhma, H., Mutanen, T., Hanski, E., Räsänen, J., Manninen, T., Lehtonen, M., Rastas, P. \& Juopperi, H. 1996. Sm$\mathrm{Nd}$ isotopic evidence for contrasting sources of the prolonged Palaeoproterozoic mafic-ultramafic magmatism in Central Finnish Lapland. IGCP Project 336 Symposium, Rovaniemi, Finland, August 21-23, 1996, Program and Abstracts. Publications of the Department of Geology and Mineralogy of the University of Turku 38, p. 17.

Hyppönen, V. 1983. Ontojoen, Hiisijärven ja Kuhmon kartta-alueiden kallioperä. Summary: Pre-Quaternary rocks of the Ontojoki, Hiisijärvi and Kuhmo map-sheet areas. Suomen geologinen kartta 1:100 000 kallioperäkarttojen selitykset lehdet 4411, 4412, 4413. 60 p.

Koistinen, T., Stephens, M. B., Bogatchev, V., Nordgulen, Ø., Wennerström, M., Korhonen, J. (comp.) 2001. Geological Map of the Fennoscandian Shield, scale 1:2 000 000, Geological Survey of Finland.

Kouvo, O. 1977. The use of mafic pegmatoids in geochronometry. In: ECOG V. Fifth European Colloquium of Geochronology, Cosmochronology and Isotope Geology, Pisa, September 5-10, 1977. 1 p.

Krogh, T. 1973. A low-contamination method for hydrothermal decomposition of zircon and extraction of $\mathrm{U}$ and $\mathrm{Pb}$ for isotopic age determinations. Geochimica et Cosmochimica Acta 37, 485-494.

Krogh, T., \& Davis, G.L. 1975. Alteration in zircons and differential dissolution of altered and metamict zircon. Carnegie Institute of Washington, Yearbook 74-75, 619-625. 
Larson, S.Å. \& Tullborg, E.-L. 2001. Why Baltic Shield zircons yield late Paleozoic, lower-intercept ages on U-Pb concordia. Geology 26, 919-922.

Lehtonen, M., Airo, M-L., Eilu, P., Hanski, E., Kortelainen, V., Lanne, E., Manninen, T., Rastas, P., Räsänen, J. \& Virransalo, P. 1998. Kittilän vihreäkivialueen geologia. Summary: The Stratigraphy, Petrology and Geochemistry of the Kittilä Greenstone Area, Northern Finland. Geological Survey of Finland, Report of Investigation 140, 1144.

Ludwig, K.R. 1991. PbDat 1.21 for MS-DOS: a computer program for processing $\mathrm{Pb}-\mathrm{U}-\mathrm{Th}$ isotope data. Version 1.08. U.S. Geological Survey Open-File Report 88-542, $35 \mathrm{p}$.

Ludwig, K.R. 2001. Users Manual for Isoplot/Ex rev. 2.49. Berkeley Geochronological Center, Special Publication No. $1 \mathrm{a}, 55 \mathrm{p}$.

Mertanen, S., Pesonen, L.J., Huhma, H. \& Leino, M.A.H. 1989. Palaeomagnetism of the Early Proterozoic layered intrusions, northern Finland. Geological Survey of Finland, Bulletin 347, 1-40.

Mezger, K. \& Krogstad, E.J. 1997. Interpretation of discordant U-Pb zircon ages: an evaluation. Journal of Metamorphic Geology 15, 127-140.

Paavola, J. 1984. Nilsiän kartta-alueen kallioperä. Summary: Pre-Quaternary rocks of the Nilsiä map-sheet area. Suomen geologinen kartta 1:100 000, kallioperäkarttojen selitykset lehti 3334, 57 p.

Papunen, H., Halkoaho, T., Tulenheimo, T. \& Liimatainen, J., 1998. Excursion to the Kuhmo Greenstone Belt. In: Hanski, E. \& Vuollo, J. (eds.) International Ophiolite Symposium and Field Excursion: Generation and Emplacement of Ophiolites through Time, August 10-15, 1998, Oulu, Finland. Abstracts, excursion guide. Geological Survey of Finland, Special Paper 26, 91-106.

Perttunen, V. \& Hanski, E. 2003. Koivun ja Törmäsjärven kartta-alueiden kallioperä. Summary: Pre-Quaternary Rocks of the Koivu and Törmäsjärvi Map Sheet Areas. Geological Map of Finland, 1 : 100 000, Explanation to the Maps of Pre-Quaternary Rocks, Sheets 3631 and 2633. Geological Survey of Finland, 88 p.

Perttunen, V. \& Vaasjoki, M. 2001. U-Pb geochronology of the Perääpohja Schist Belt, northwestern Finland. In: Vaasjoki, M. (ed.) Radiometric Age Determinations from Finnish Lapland and Their Bearing on the Timing of Precambrian Volcano-Sedimentary Sequences. Geological Survey of Finland, Special Paper 33, 45-84.

Pigeon, R.T., O’Neil, J.R. \& Silver, R.T. 1995. The interdependence of U-Pb stability, crystallinity and external conditions in natural zircons - an early experimental study. Leon T. Silver $70^{\text {th }}$ Birthday Symposium and Celebration, Extended Abstracts, p. 225-231.

Pidgeon, R.T., O’Neil, J.R. \& Silver, L.T. 1973. Observations on the crystallinity and the U-Pb system of a metamict
Ceylon zircon under experimental hydrothermal conditions. Fortschritte in der Mineralogie 50, 118.

Piirainen, T. 1969. Initiale Magmatismus und seine Erzbildung in der Beleuchtung des Koli-Kalimogebietes. Bulletin of the Geological Society of Finland 41, 21-45.

Piirainen, T. \& Vuollo, J. (eds.) 1991. Arkeeinen ja proterotsooinen geologinen evoluutio ja malminmuodostus. Pohjois-Karjalan malmiprojektin loppuraportti. Oulun yliopisto, Pohjois-Karjalan Malmiprojekti, Raportti 31, $145 \mathrm{p}$.

Räsänen, J. \& Huhma, H. 2001. U-Pb datings in the Sodankylä schist area of the Central Lapland Greenstone Belt. In: Vaasjoki, M. (ed.) Radiometric Age Determinations from Finnish Lapland and Their Bearing on the Timing of Precambrian Volcano-Sedimentary Sequences. Geological Survey of Finland, Special Paper 33, 153-188.

Richard, P., Shimizu, N. \& Allègre, C.J. $1976 .{ }^{143} \mathrm{Nd} /{ }^{146} \mathrm{Nd}$, a natural tracer: an application to oceanic basalts. Earth and Planetary Science Letters 31, 269-278.

Sakko, M. 1971. Varhais-karjalaisten metadiabaasien radiometrisiä zirkoni-ikiä. Summary: Radiometric zircon ages on the Early-Karelian metadiabases. Geologi 23, 117-119.

Stacey, J.S. \& Kramers, J.D. 1975. Approximation of terrestrial lead isotope evolution by a two-stage model. Earth and Planetary Science Letters 26, 207-221.

Tulenheimo, T. 1999. Kuhmon Kellojärven kerroksellinen ultramafinen muodostuma. Unpublished M.Sc. Thesis, University of Turku, 199 p. (in Finnish).

Tyrväinen, A. 1983. Sodankylän ja Sattasen kartta-alueiden kallioperä. Summary: Pre-Quaternary Rocks of the Sodankylä and Sattanen Map Sheet Areas. Geological map of Finland 1:100 000, Explanation to the Maps of PreQuaternary Rocks, Sheets 3713 and 3714. Geological Survey of Finland, 59 p.

Vuollo, J. \& Huhma, H. 2005. Paleoproterozoic mafic dikes in NE Finland. In: Lehtinen, M., Nurmi, P.A. \& Rämö, O.T. (eds.) Precambrian Geology of Finland. Key to the Evolution of the Fennoscandian Shield. Elsevier Science B.V., Amsterdam, pp. 193-235.

Vuollo, J. \& Piirainen, T. 1992. The 2.2 Ga old Koli layered sill: The low-Al tholeiitic (karjalitic) magma type and its differentiation in northern Karelia, eastern Finland. Geologiska Föreningens i Stockholm Förhandlingar 114, 131142.

Wasserburg, G.J., Jacobsen, S.B., DePaolo, D.J., McCulloch, M.T. \& Wen, T. 1981. Precise determination on Sm/Nd ratios, $\mathrm{Sm}$ and $\mathrm{Nd}$ isotopic abundances in standard solutions. Geochimica et Cosmochimica Acta 45, 2311-2323.

Whitehouse, M., Claesson, S., Sunde, T. \& Vestin, J. 1997. Ion microprobe $\mathrm{U}-\mathrm{Pb}$ zircon geochronology and correlation of Archaean gneisses from the Lewisian Complex of Gruinard Bay, northwestern Scotland. Geochimica et Cosmochimica Acta 61, 4429-4438.

Whitehouse, M.J., Kamber, B.S. \& Moorbath, S. 1999. Age 
significance of U-Th-Pb zircon data from early Archaean rocks of west Greenland - a reassessment based on combined ion microprobe and imaging studies. Chemical Geology 160, 201-224.

Wiedenbeck, M., Allé, P., Corfu, F., Griffin, W.L., Meier, M., Oberli, F., von Quadt, A., Roddick, J.C., \& Spiegel, W. 1995. Three natural zircon standards for U-Th-Pb, Lu-
Hf, trace element and REE analysis. Geostandards Newsletter 19, 1-23.

Williams, I.S. 1998. U-Th-Pb geochronology by ion microprobe. In: McKibben, M.A., Shanks, W.C., III \& Ridley, W.I. (eds.) Applications of Microanalytical Techniques to Understanding Mineralizing Processes. Reviews in Economic Geology 7, 1-35. 